\title{
Magnetic Field Considerations in Fusion Power Plant Environs
}
by
H. B. Liemohn
D. L. Lessor
B. H. Duane

September 1976

Prepared for the Energy Research and Development Administration under Contract E(45-1)-1830

\section{\%ัต Battelle}


NOTICE

This report was prepared as an account of work sponsored by the United States Government. Neither the United States nor the Energy Research and Development Administration, nor any of their employees, nor any of their contractors, subcontractors, or their employees, makes any warranty, express or implied, or assumes any legal liability or responsibility for the accuracy, completeness or usefulness of any imformation, apparatus, product or process disclosed, or represents that its use would not infringe privately owned rights.

\author{
PACIFIC NORTHWEST LABORATORY \\ operated by \\ BATTELLE \\ for the
}

ENERGY RESEARCH AND DEVELOPMENT ADMINISTRATION

Under Contract E(45-1)-1830

Printed in the United States of America

$$
\text { Available from }
$$

National Technical Information Service

U.S. Department of Commerce

5285 Port Royal Road

Springfield, Virginia 22151

Price: Printed Copy \$5.50; Microfiche $\$ 2.25$ 
BNWL-2021

UC -20

\title{
33679000624702
}

MAGNETIC FIELD CONSIDERATIONS

IN FUSION POWER PLANT ENVIRONS

\author{
by \\ H. B. Liemohn \\ D. L. Lessor \\ B. H. Duane
}

September 1976

BATTELLE

Pacific Northwest Laboratories Richland, Washington 99352 


\section{PREFACE}

Fusion reactor technology has developed far enough to expect laboratory demonstration of practical levels of fusion employing the D-T reaction to occur in the early 1980s. Following that demonstration, and depending upon the national priorities for energy from D-T fusion, construction and operation of experimental reactors and demonstration power reactors could occur before the end of this century. Operation of the first commercial power plants could then follow, starting about 2010 .

Development and adoption of a new power system eventually will require a description of the environmental effects in an environmental statement providing a comparison to the effects of competitive systems. In anticipation of that statement, an environmental analysis (BNWL-2010) has been prepared for the ERDA Division of Magnetic Fusion Energy. That analysis estimates the environmental effects of constructing and operating D-T fusion reactors as an economically competitive source of electricity in the 21 st century.

The analysis has four primary purposes:

1. To describe the general nature of the environmental effects,

2. To determine current ability to estimate the effects,

3. To determine methods for reducing the effects, and

4. To determine research necessary for increasing capability to define and reduce the effects.

Timely identification of needed research and methods for reducing effects will permit the performance of that research and the revision of conceptual fusion power plant designs before preparation of the program environmental statement. This would improve the quality of the environmental statements and could reduce the estimated adverse environmental effects due to fusion power plants.

The environmental analysis (BNWL-2010) concludes that the following assumed characteristics are the best set for the first operating fusion power plants:

- The D-T fusion reaction

- Large quantities of activation products

- Kilogram quantities of tritium in the plant systems

- Massive reactor structures

- Large lithium inventories

- Large inventories of liquid metals and salts
- Standard electricity generation

- Standard radioactive waste systems

- Large magnetic fields

- A self-contained fuel cycle

- Rural siting

Using these characteristics a reference reactor was analyzed to determine the environmental effects by using available concepts of plant subsystems designs that control interactions with the environment or by assumption that best current technology would be used in subsystems design.

Because this analysis does not take into account advances in both fusion and waste control technology during the next thirty years, the estimated effects probably are significantly higher than the actual effects will be for the first fusion power plants. The estimated environmental effects should be interpreted only as being the probable upper limit for the actual effects. 
Preparation of the fusion power plant environmental analysis required development and use of specially developed data and analysis methods not used in the preparation of current environmental statements for fossil and fission power plants. These data and analysis requirements are documented in a series of reference topical reports to make this information publicly available and to assure understanding of the basis for the conclusions made in the environmental analysis. These reference topical reports summarize the state-of-the-art as applicable to preparation of environmental statements for fusion power plants. They present the data and analytical techniques used in the environmental analysis to estimate the interactions with the environment and the resultant environmental effects. This information then was analyzed for adequacy and the need was determined for additional research to assure satisfactory ability to prepare environmental statements for the fusion development program and experimental facilities in the early 1980s. Estimated environmental effects are presented in these reference documents only as necessary to illustrate use of the data and analytical techniques.

This report is one of those reference documents for the environmental analysis. The other documents in this series contain more details of the power plant concepts and the probable environmental effects of fusion power plants with the assumed characteristics listed above. These documents are available through the National Technical Information Service:

An Environmental Analysis of Fusion Power to Determine Related R\&D Needs, BNWL-2010

Review of Fusion Research Program: Historical Summary and Program Projections, BNWL-201]

Fuel Procurement for First Generation Fusion Power Plants, BNWL-2012

Current Fusion Power Plant Design Concepts, BNWL-2013

Reference Commerical Fusion Power Plants, BNWL-2014

Siting Commercial Fusion Power Plants, BNWL-2015

Materials Availability for Fusion Power Plant Construction, BNWL-2016

Projected Thermodynamic Efficiencies of Fusion Power Plants, BNWL-2017

Tritium Source Terms for Fusion Power Plants, BNWL-2018

Management of Nontritium Radioactive Wastes from Fusion Power Plants, BNWL-2019

Methodology for Estimating Radiation Doses Due to Tritium and Radiocarbon Releases, BNWL-2020

Magnetic Field Considerations in Fusion Power Plant Environs, BNWL-2021

Biological Effects of Tritium Releases from Fusion Power Plants, BNWL-2022

Biological Effects of Activation Products and Other Chemicals Released from Fusion Power Plants, BNWL-2023

Safety Review of Conceptual Fusion Power Plants, BNWL-2024

An Investigation of the Transportation Requirements of Fusion Power Plants, BNWL-2025

Considerations of the Social Impact of Fusion Power, BNWL-2026

Environmental Impacts of Nonfusion Power Systems, BNWL-2027

Environmental Cost/Benefit Analysis for Fusion Power Plants, BNWL-2028

Biomagnetic Effects: A Consideration in Fusion Reactor Development, BNWL-1973

An Analysis of Tritium Releases to the Atmosphere by a CTR, BNWL-1938 
CONTENTS

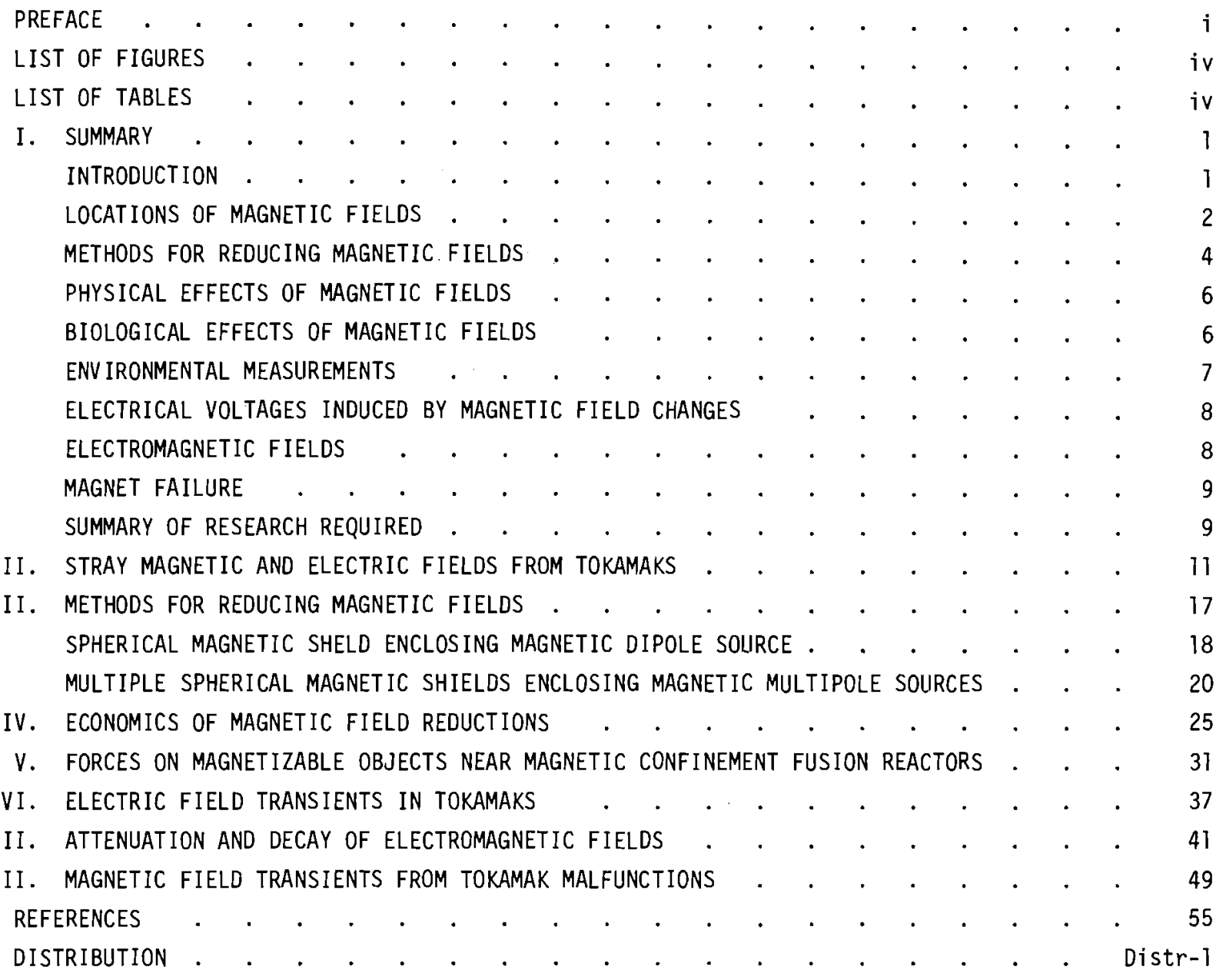




\section{$\underline{\text { LIST OF FIGURES }}$}

Behavior of Stray Magnetic Fields . . . . . . . . . . . . . . . 3

Cross Section View of UWMAK-I Fusion Reactor . . . . . . . . . . . 12

UWMAK-I Transformer and Divertor Currents . . . . . . . . . . . . 13

4 Magnetic Flux Surfaces, Divertors and Coils in PPPL Tokamak Design . . . . . 13

5 Cross Section of the Nuclear Island in PPPL Tokamak Design . . . . . . . 14

6 Magnetic Field Magnitude Contours in UWMAK-I Midplane . . . . . . . . . 15

7 Magnetic Field Magnitude Contours in Vertical Plant for UWMAK-I . . . . . 15

8 Near Magnetic Field Magnitude Contours in Vertical Plane for UWMAK-I . . . . 16

9 Spherical Magnetic Shield Enclosing Magnetic Dipole Source . . . . . . . 18

10 Concentric Spherical Shell Shields Enclosing Magnetic Multipole Sources . . . . 20

11 Ratio of Magnetic Force to Gravitational Force on a Long, Slender, Magnetic . . . . 34

12 Contours of Constant Ratio of Magnetic Force $F_{M}$ To Gravitational Force $F_{G}$ on Long

\section{$\underline{\text { LIST OF TABLES }}$}

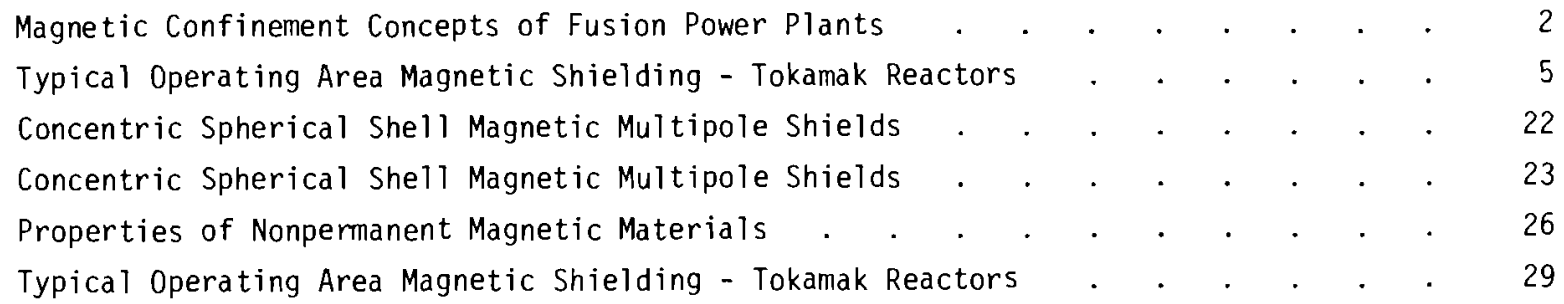


I. SUMMARY

H. B. Liemohn

\section{INTRODUCTION}

Large magnetic fields to confine the hot plasma fuel are required for many concepts for fusion power plants. Inherently, these fields extend beyond the immediate vicinity of the reactor and thus represent a potential impact on the surrounding environment. Various aspects of the external stray magnetic fields discussed in this report include spatial extent, methods for reducing their strength, important physical effects, possible biological concerns, induced electric fields, and electromagnetic radiation.

Magnetic phenomena are believed to arise from forces between electric charges in motion. Magnetic fields are generated by electric current that is driven through circuitry or inherently present inside magnetic materials. Steady fields produce forces on other moving electrical charges such as currents in wires, individual particles in plasmas, or atomic structure in magnetic materials. Changing magnetic fields induce currents in other conducting materials by generating transient electric fields. These properties enable magnetic fields to confine and energize the fusion material which consists of charged particles called plasma.

In fusion reactors where plasma fuel must be confined and controlled, the desired magnetic fields are generated by large arrays of current coils. The new technologies required for creation of such large-scale and high intensity magnetic fields pose both control problems and environmental questions that need further analysis. Our purpose is to identify those magnetic field characteristics that have environmental implications and assess their relative significance.

An important inherent property of magnetic fields is their monotonic decrease away from source regions. Thus, at sufficiently large distances from fusion reactors, the magnetic field is reduced to a level comparable to the surface magnetic field of the earth $(0.3$ gauss at the equator to 0.7 gauss near the poles). In general this far-field decreases at least as fast as the inverse cube of distance from the source region; however, the field near the source may vary quite differently. The shape and magnitude of the far field are estimated in this report for various reactor configurations.

Reduction of stray magnetic fields can be achieved in two ways. One way is to shield the field with magnetic material such as iron or special alloys. These materials alter the field by establishing a preferred path for the imposed magnetic field. The second method is to introduce another source of magnetic field such as current coils that create an opposing field. Both methods can virtually el iminate magnetic effects on local regions. However, such shielding affects the shape and magnitude of the primary field in the reactor and must be included in the engineering design. 
Physical effects of magnetic fields on objects are fairly well understood. Electronic devices are particularly susceptible to interference and the structural metal in the building is also affected. Detailed studies must await specific reactor designs but some general considerations are possible.

Biological effects of high magnetic fields are relatively unknown at this time. If experiments show adverse effects from prolonged exposure to above normal fields, appropriate steps should be taken to avoid exposure. Solutions might include moving routine access limits for plant personnel and plant boundaries greater distances from the reactor, reducing exposure time in such fields, and building plant operation consoles either at a distance or inside local bucking coil configurations. The entire reactor could be enclosed in magnetic material but this is unlikely because of the mass of material involved. However, local shielding of operating personnel and instrumentation is feasible.

Changing magnetic fields in fusion reactors induce transient electric fields that must be considered. These fields are like battery potentials that drive current in the reactor's conducting elements that link the changing magnetic flux. They must be considered in maintenance procedures. Preliminary estimates based on present reactor operation concepts show that these electric fields are very small outside the main hall of the reactor.

Rapid variations of magnetic fields, switching of large currents, and plasma particle accelerations produce copious amounts of electromagnetic (microwave) radiation. Very thin walls of conducting material are capable of stopping such energy. Thus, the containment vessel of the plasma fuel and conducting cages around other sources should eliminate this hazard for operating personnel, and external radio and television interference should be nil.

\section{LOCATIONS OF MAGNETIC FIELDS}

The configuration and magnitude of the magnetic field within and around a fusion power reactor depends on the specific design. Table 1 lists the present concepts and the originating laboratory. All fusion concepts that require plasma confinement employ very large magnetic fields (up to 160,000 gauss internally has been proposed). Some designs operate in an almost steady-state mode while others use repetitive firing at a high rate. All configurations have startup and shutdown procedures when significant magnetic field changes occur that induce large electric fields. We are concerned in this subsection with the large slowly varying external magnetic fields, and induced electric fields from rapid magnetic variations during normal operations are discussed in subsequent subsections.

TABLE 1 Magnetic Confinement Concepts of Fusion Power Plants

\begin{tabular}{ll}
\multicolumn{1}{c}{ Design } & \multicolumn{1}{c}{ Originating Laboratory } \\
Tokamak & University of Wisconsin (UWMAK) \\
& Brookhaven National Laboratory (BNL) \\
& Princeton Plasma Physics Laboratory (PPPL) \\
& Oak Ridge National Laboratory (ORNL) \\
& Los Alamos Scientific Laboratory (LASL) \\
Theta Pinch & Lawrence Livermore Laboratory (LLL)
\end{tabular}


The present tokamak designs generate a slowly varying external dipole field which is produced by the poloidal field coils that maintain the plasma. The steady-state toroidal fields are actually much larger internally but their external field cancels more effectively. A more detailed discussion of tokamak fields is presented in Section II. The yin yang (baseball seam coil) configuration also has a strong external field that is predominantly dipolar. The field strength of a dipole falls off inversely with the cube of distance. This rapid fall-off of stray fields from these reactors is illustrated in Figure 1. At a distance of approximately 50 meters from the center of these reactors, where the biological shield for radiation confinement will probably be located, the steady magnetic field is several hundred gauss during normal operation. At several hundred meters without magnetic shielding, the external field becomes comparable to the geomagnetic background of a few tenths of a gauss.

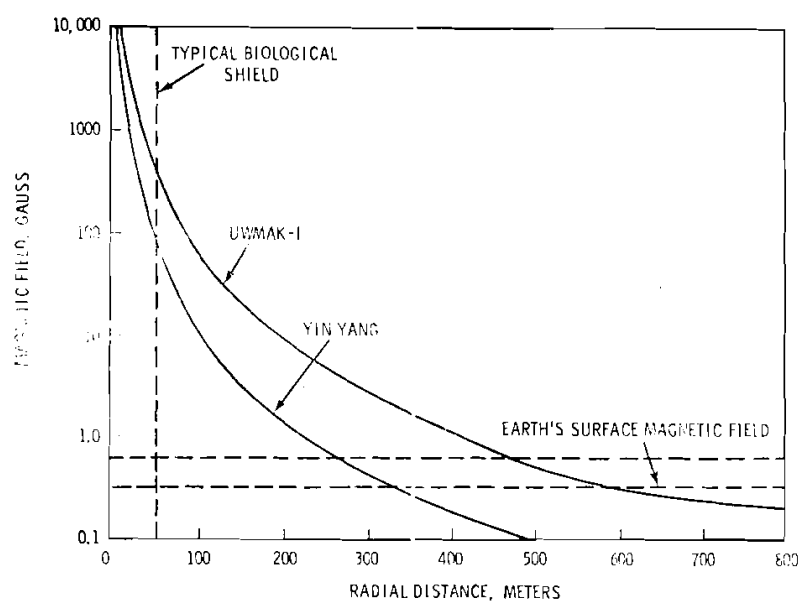

FIGURE 1 Behavior of Stray Magnetic Fields

Although the internal confinement field of the theta-pinch reactor is comparable to other designs, its external magnetic field is probably smaller than that of tokamaks due to effective cancellation of opposing field coil elements, but no preliminary design estimates are available. The large diameter of the theta-pinch reactor (112 meters) prevents accurate approximations from being used inside a few ring diameters where personnel would normally operate. Another aspect of this design is its rapid repetitive firing which induces large electric field transients and produces significant electromagnetic radiation. A more detailed assessment of stray magnetic and electric fields from theta-pinch devices will be needed as designs evolve, but rapid falloff with distance is anticipated. 
The laser implosion concept does not employ large magnetic fields; only a modest divertor field is needed to protect its inner wall. Consequently, its magnetic aspects are not considered here.

As part of the environmental research program, it is advisable to measure the magnitude and configuration of the external magnetic fields around existing large-scale plasma confinement devices. Such measurements would provide an empirical basis for estimating the external fields of future power reactors. Near-field configurations would provide valuable data for the effective multipole structure of the source region. Measurements around reinforced concrete walls and other permeable structures would help define the effect of reactor hall buildings on the magnetic field.

Measurements of the magnetic environment around selected currently operating experiments would also provide a basis for estimating the total magnetic flux exposure of experimenters. From the operations $\log$ it might be possible to reconstmet the gauss-hour exposure of personnel. This medical history would significantly enlarge the statistical data base for investigations of possible biomagnetic effects.

\section{METHODS FOR REDUCING MAGNETIC FIELDS}

The stray magnetic fields around fusion reactors can be sharply reduced by appropriate configurations of permeable materials. Both common ferromagnetic materials and high permeability alloys have potential advantages and disadvantages. Present reactor designs do not incorporate any magnetic material since its distortion of confinement geometries is difficult (and expensive computationally) to evaluate. Eventually, the perturbing effects of permeable materials such as reinforcing iron rods in the reactor foundation and wall structure will have to be considered in the design.

In order to assess the attenuation of steady-state magnetic fields by walls of high permeable material, a specific quantitative model was solved analytically in Section III. An entire fusion reactor represented as a magnetic dipole was enclosed in a spherical shell of highly permeable material. Such a shell traps magnetic flux provided that the field strength does not exceed saturation. As an example, for dipole strengths appropriate to fusion reactors, the leakage field can be reduced to that of the natural environment by a pure iron shell about 3 meters thick with an outer radius near 15 meters. Since the permeability and saturation level of magnetic elements and alloys differ markedly, a multilayer shield may be more effective.

A theoretical analysis assessing the economics of magnetic shielding is presented in Section IV. Variations in shel1 thickness, location, and material properties have been considered as tradeoffs. The calculations showed that reducing the magnetic field strength by a factor of 100 around a reactor would require a spherical iron shel1 1.8 meters thick at a radius of 50 meters with a weight of 440,000 metric tons. Therefore, reduction of stray magnetic fields to the level of the earth's field at the biological shield ( 50 meters) does not seem economically feasible. However, shielding a control room with permeable material is feasible from an economic and resource utilization standpoint. Table 2 presents the shielding requirements for 
6 meter diameter rooms at various distances from a typical reactor. The presence of large permeable shields would alter the local plasma confinement fields within the reactor if the coil current is not changed. Another consideration is the hysteresis, or magnetic memory, of magnetic materials in and around the fusion reactor. A significant amount of residual magnetism in structural walls or mechanical controls may affect maintenance procedures.

TABLE 2 Typical Operating Area Magnetic Shielding - Tokamak Reactors

\begin{tabular}{|c|c|c|c|c|}
\hline \multirow[b]{2}{*}{$\begin{array}{l}\text { Distance from } \\
\text { Reactor Center } \\
\text { (meters) }\end{array}$} & \multirow{2}{*}{$\begin{array}{l}\text { Magnetic Field } \\
\text { Strength Without } \\
\text { Shielding } \\
\text { (gauss) }\end{array}$} & \multicolumn{3}{|c|}{ She11 Requirements } \\
\hline & & $\begin{array}{l}\text { Vol ume } \\
\text { (meter }^{3} \text { ) }\end{array}$ & $\begin{array}{l}\text { Mass } \\
(\mathrm{kg})\end{array}$ & $\begin{array}{c}\text { Cost } \\
(\$ \text { at } \\
\$ 4.40 / \mathrm{kg}) \\
\end{array}$ \\
\hline 50 & 550 & 14 & 110,000 & 480,000 \\
\hline 100 & 70 & 1.6 & 12,000 & 54,000 \\
\hline 150 & 20 & 0.47 & 3,700 & 16,000 \\
\hline 200 & 9 & 0.20 & 1,600 & 7,000 \\
\hline 325 & 2 & 0.05 & 370 & 1,600 \\
\hline
\end{tabular}

Basis: Spherical shell, 3 meter inner radius, 1 gauss inner magnetic field, shell relative permeability of 200,000 , magnetic saturation of 1,500 gauss.

The steady magnetic field can be virtually eliminated in local regions by means of bucking coils which avoid the adverse effect of hysteresis. Such coils merely produce a local field of opposite polarity that approximately cancels the local stray field of the reactor. Everything from miniaturized electronic circuits to modest-size control rooms for operating personnel might be effectively shielded in this manner. Since the shielding field must not alter plasma confinement, its effect must be included in the overall design, or the local region must be sufficiently removed from the plasma that the bucking coil fields are relatively negligible in the reactor.

Shielding stray electromagnetic fields produced by sharply pulsed or rapidly varying currents in normal reactor operation appears feasible. Details of the electromagnetic spectrum radiated by non-tokamak reactors that recycle rapidly have not been specified. However, present state-ofthe-art electrical engineering techniques are believed to provide adequate attenuation. Even the extreme case of a lightning-flash collapse of superconductivity in the main confinement coils may be effectively shielded. It may require a thin copper shell at the biological shield or perhaps the structural steel (or iron) in the walls may be sufficient. Appropriate criteria can be incorporated in the engineering design.

The physical principles for local reduction of magnetic fields are well established and readily applied as necessary. Thus, there is no apparent need for further engineering research in this area at the present time, although three-dimensional applications are extremely difficult. The design of prototype reactors should include some provision for shielding and local field reduction, however, to ascertain realistic cost estimates for production power reactors. 


\section{PHYSICAL EFFECTS OF MAGNETIC FIELDS}

Large magnetic fields and electromagnetic signals generated in the operation of a fusion reactor interact with the surrounding instrumentation and structural materials. Their sensitivity to such fields varies widely and must be taken into account by the engineering design. The purpose of this subsection is to consider some important general questions on the physical effects where more research is needed.

The steady magnetic field external to the reactor may affect the operation of electronic components. For some applications the background field may become comparable to that in inductive elements of miniaturized circuitry, and magnetic forces may alter the conduction properties of certain solid state devices. Circuitry in moving apparatus or portable equipment may also be affected by induced electric fields and currents. The electronic control systems that are required in the reactor hall should be tested for sensitivity to large magnetic fields. Methods for effective magnetic shielding should be identified and tested as well.

Some magnetic materials will probabiy be used in the construction of fusion reactor buildings. These materials may be readily apparent as mechanical fittings or supporting elements within the reactor building, or they may be buried as reinforcing iron rod within the walls and structural members of the building. Such ferromagnetic structural materials will develop some residual magnetism due to hysteresis, but it is not expected to be of any consequence in the structural integrity or the environment. This hysteresis effect might be important, however, if maintenance personnel must enter areas during a shutdown after the structure has been subjected to intense fields. These fields may also stimulate corrosion and fracture in certain materials over very long time spans, but these effects are probably significant only within the reactor itself. Further research on maynetic stresses in structural materials is advisable to assure long-term reliability.

Magnetic forces on magnetitized objects can be very large in regions where intense magnetic fields have spatial variation in field strength. In a dipole field whose strength is 500 gauss at 50 meters out in mid-plane ( imilar to UWMAK-I), magnetic force on isolated iron objects can exceed gravitational inside about 25 meters and drop to about 10 percent of gravitational at about 45 meters. For a more detailed account, see Section $V$. An annoyance and some hazard would accompany use of magnetizable tools and other objects in such close proximity because of the magnetic interaction between them. Use of non-magnetizable tools will largely eliminate the problem. Workmen will typically be excluded from magnetic field regions of more than 500 gauss during operation because of ionizing radiation.

\section{BIOLOGICAL EFFECTS OF MAGNETIC FIELDS}

While there exist uncertainties in magnitude of both the currents and the physical dimensions in an eventual fusion power reactor, one anticipates that plant personnel will receive continuing exposure to magnetic fields of a few hundred gauss. Offsite personnel will routinely enter a region of 1 to a few tens of gauss near the plant boundary. 
In general, the biological effects of chronic exposures to magnetic fields are not known. It would appear that biological experiments to assess tokamak fusion reactor magnetic field impact should consider:

- Continued exposure of living organisms to up to 500 gauss fields, to which operator and maintenance people may be exposed,

- Occasional exposure of nonhwman living organisms to up to 500 gauss fields, as may occur to overflying birds,

- Both continued and occasional exposure to about 1 gauss fields differing in direction from the earth's, as may happen to persons and animals living near or passing by the plant boundaries.

The effects one might seek experimentally are: long-term physiological problems from continued exposure to 500 gauss fields and short-term illness, e.g., headaches from exposure at this level; subtle behavioral effects, e.g., disorientation for living organisms at the 500 gauss level; and a role for ambient magnetic field direction in normal biological functions like sense of direction.

Biological effects are reviewed in "Biomagnetic Effects: A Consideration in Fusion Reactor Development". (1) The studies that have been reported have not obtained definitive results due to inadequate statistical bases, discrepancies in conclusions, and nonreproducibility. While many studies report no detectable biological effects for exposure to high intensity fields, others found measurable changes after exposure to relatively small fields. As a consequence of these ambiguous results, further research is recommended. Several explicit problem areas are suggested for study by D. D. Mahlwo. (1)

\section{ENVIRONMENTAL MEASUREMENTS}

The personnel who operate fusion reactors should monitor their instantaneous and total exposure to the magnetic field environment in a manner analogous to that now used for nuclear radiation. However, such instrumentation is not available. Several different instruments are needed to measure the instantaneous local field, accumulated magnetic-flux exposure, the local rate of change of field, accumulated electric potential exposure, and finally microwave exposure. Laboratory instruments are currently available to measure all of these functions; however, personal dosimeters need to be developed.

Research should begin immediately to obtain the technology for the construction of personal dosimeters for magnetic exposure. Since fusion researchers are using large magnetic fields in their current experimental investigations, their working environment should be monitored routinely for stray fields. This would provide an important data base for future studies of biological implications. Thus, development of dosimeters should have high priority. 


\section{ELECTRICAL VOLTAGES INOUCED BY MAGNETIC FIELD CHANGES}

In the normal operation of fusion reactors, their magnetic fields are changed quite rapidly. For tokamak designs the poloidal field recycles at the end of each burn period, whereas the theta-pinch fields are pulsed repetitively at a high rate. Such changing magnetic fields induce electric fields around the reactor, which may drive currents or build up charges in conductors. Based on the projected field magnitudes and rates of change, these electric fields are not expected to be a hazard to personnel outside of the reactor hal1. A more complete description of induced field strengths is given in Section VI.

Inside the biological shields for large tokamaks the electric fields are estimated to be about 3 volts per meter at a radius of 12 meters around the reactor. A wire that encircles the reactor could have an induced voltage of as much as 200 volts between two ends. Proper grounding of all conducting materials near a reactor should prevent electrical hazards for both personnel and equipment. Appropriate safety procedures will be necessary during maintenance and operating activities requiring alteration of grounding systems or separation of equipment items from their normal grounding systems. No new research is indicated in this area.

\section{ELECTROMAGNETIC FIELDS}

Rapidly varying fields occur in fusion reactors during their continuous operation as well as during startup and shutdown. Both the transient electric potentials induced by rapidly changing magnetic fields and electromagnetic radiation have been considered. The proposed reactors operate on a duty cycle for the fusion burn that ranges from milliseconds in laser and theta-pinch concepts to an hour or more for tokamaks and yin yang designs. Rapid current switching among reactive circuit elements generates substantial microwave noise. Thus there is a broad range of rates for magnetic field change and a wider range of radiated frequencies. Some aspects of the attenuation and decay of electromagnetic fields in tokamaks are presented in Section VII.

Induced electric potentials are small in the normal operating cycle. Although the magnetic fields are large in tokamaks, their rate of change is so slow that electric fields of less than one volt per meter are anticipated at the biological shield. The much more rapid rate of change required in the theta-pinch is compensated by the high-order multipole moment, so that the potentials are similarly negligible beyond its biological shield.

Electromagnetic energy that is generated in the fusion burn is confined entirely within the reactor. Most of this wave energy is reflected back into the plasma by the conducting walls of the containment vessel and the rest is absorbed as heat. Other electromagnetic energy generated in the reactor room is expected to be completely trapped inside the biological shield so that operating personnel are protected. Nevertheless, its effects inside the shield should be considered. For example, electric potentials induced by rapidly changing magnetic fields could generate undesirable charges on isolated conducting material. Cycling of fields also causes eddy currents in mechanical and electronic controls that may be important over the lifetime of a reactor. Research on structural fatigue is advocated in the prototype reactor design staye. 
The electrical switching network associated with fusion reactor operation generated copious amounts of microwave energy. The superconducting coils are coupled inductively so large back electromotive forces occur in the course of rapidly switching large currents. Technological improvements are steadily reducing this loss of energy and a conducting cage readily confines microwaves. Thus, this aspect of reactor operation poses no unusual environmental problems and is not expected to cause harm to plant operating personnel if standard industrial safety practices are used.

\section{MAGNET FAILURE}

The accidental release of large amounts of energy stored in reactor superconductors must be evaluated. A sudden cryogenic failure could cause one or more coils to lose superconductivity. Since the electrical current is inductively coupled to other coils, part of the energy is transferred through their mutual fields; the remainder dissipates as heat in the nonsuperconducting coil material. The energy dissipation may heat the coil only a few hundred degrees, at most, assuming a uniform distribution throughout the conductor. Coil designs must avoid localized dissipation that could have explosive consequences.

Such an accident creates abnormal magnetic field configurations in the immediate vicinity of the reactor. For example, the loss of one toroidal field coil in the tokamak allows some added field leakage but it would not significantly increase the magnetic field at the biological shield ( 250 meters). The actual shape of the field distortion depends on the machine configuration and the number of affected coils. A more important consideration is the resulting mechanical stresses that swell the laminated superconductor and may necessitate its replacement.

A rapidly changing field distortion due to coil failure induces large electric potentials that need to be evaluated. Normally compensating changes in other superconductor coil currents would 7 imit the growth of stray electric fields. However, since the superconductor coils are expected to operate near their saturation field cutoff for cost effectiveness, the additional current load in adjacent coils could exceed saturation and revert them to normal conduction as well. The resulting cascade would generate a very high local electric field that would manifest itself in arc discharges and large electromagnetic wave amplitudes. The magnitude of the electric fields inside the biological shield depends on such factors as the magnetic field configurations, the rate of field change, and the distribution of conducting materials.

Quantitative estimates of the field transient from tokamak malfunctions are described in Section VIII; but a detailed quantitative study is recommended. Research should begin now on failure modes in order to incorporate appropriate safety controls in the prototype designs.

SUMMARY OF RESEARCH REQUIRED

Magnetic fields may have adverse biological effects on power plant operating personnel and other forms of life entering the power plant exclusion areas. The following types of research should be started immediately to obtain as much additional information as possible prior to preparation 
of the first program and experimental power reactor environmental statements expected early in the 1980s. This information is needed to either provide assurance that magnetic fields do not have adverse effects or to identify methods for minimizing any adverse effects.

- Measurement of the external magnetic fields around existing large-scale plasma confinement devices to better estimate external fields of future fusion reactors.

- Measurement of local field distortions by reinforced concrete walls and other permeable structures around experimental plasma confinement devices.

- Measurement of the stray field environment around operator consoles of existing largescale plasma confinement devices to study possible biomagnetic effects to personnel.

- Provision in prototype designs for shielding and local field reduction to ascertain realistic cost estimates for production power reactors.

- Study of magnetic stresses in structural materials.

- Development of a personal dosimeter to monitor magnetic field exposure of fusion researchers and future reactor operators.

- Study of failure mode physics to assist prototype designers in incorporating appropriate safety controls in the magnetic coils.

- Determination of biological effects of magnetic fields. 


\title{
II. STRAY MAGNETIC AND ELECTRIC FIELDS FROM TOKAMAKS
}

\author{
D. L. Lessor
}

The slowly varying stray magnetic field in the vicinity of a tokamak power plant comes from two types of current geometry: 1) toroidal currents, including those in the plasma itself, in poloidal field transformer coils, in vertical and control field coils, and in the divertors, and 2) the poloidal currents in the toroidal field coils ("D's"). The primary source of the slowly varying magnetic field, at a distance large compared to the major radius of the toroid, is predominantly the poloidal dipole field of the transformer coils, which is inversely proportional in magnitude to the cube of the distance from the reactor center. The toroidal field of the $D$ assembly is much larger internally but they are arrayed circumferentially equidistant about the torus with diametrically opposite coils oppositely oriented, and in normal operation their strong field contribution is only significant within a few field coil diameters.

Magnetic field calculations have been performed for the tokamak reactor design studies at the University of Wisconsin and at Princeton Plasma Physics Laboratory. A study of the stray magnetic field in the UWMAK-I Wisconsin design is reported by T. F. Yang, et al. (2) Yang calculated the magnetic fields from a collection of filamentary circular current rings, with either 12 or 24 current loops located symmetrically about the torus to represent the toroidal field source currents. The toroidal currents generating the poloidal fields were similarly represented by circular current rings centered on the torus symmetry axis in a separate calculation. This representation of the field is adequate for calculation of magnetic field everywhere except at distances from the coils that are small compared with transverse dimensions of the nearest current flow areas. The calculation was a Biot-Savart law type calculation with no magnetic materials present.

Magnetic materials probably will not be used in the most intense field regions of tokamak reactors, since field strengths will exceed their magnetic saturation. But it might be desirable to use magnetic materials in less intense field regions for field shaping or local shielding. Contemporary research tokamaks do use magnetic poloidal field transformer cores.

The poloidal magnetic dipole field contribution from the transformer coils is systematically varied with time during the tokamak reactor operating cycle. UWMAK-I ${ }^{(3)}$ and $\mathrm{PPPL}^{(4)}$ design employed 96.5 minute and 100 minute operating cycles, respectively. Throughout the burn period, the poloidal field coil current is systematically changed to maintain a nearly constant plasma current. The distant magnetic field uuring the burn cycle has its source strength determined by the sum of the protucts of toroidal currents times the area of the current loop, and will therefore show some variation during the burn cycle. The major toroidal currents in UWMAK-I are shown in Figures 2 and 3 reproduced from the UWMAK-I document. (3) The tabulation summarizes the leading divertor current contributions and the transformer current ranges. Even though the transformer currents are of comparable magnitude to the divertor currents and the plasma current, their contribution to the dipole field is smaller because of their smaller areas. The dipole 
field calculated by Yang is therefore representative of must of the burn cycle. Placing divertor cills inside the $D^{\prime}$ 's could allow some reduction in divertor current and resulting dipole field but the reduction is likely to be on the order of a factor of two. Yang's calculation assumed a toroidal plasma current of 26.2 megamperes, compared with an operating current of 20.6 megamperes assumed in the UWMAK-I design. Variations of this order are expected in tokamak fusion reactor designs. The Princeton design had a plasma current of 14. 6 megämperes and lower divertor currents inside turnidal field coils, as shown in Figures 4 and 5 reproduced from the Princeton tokamak design document.

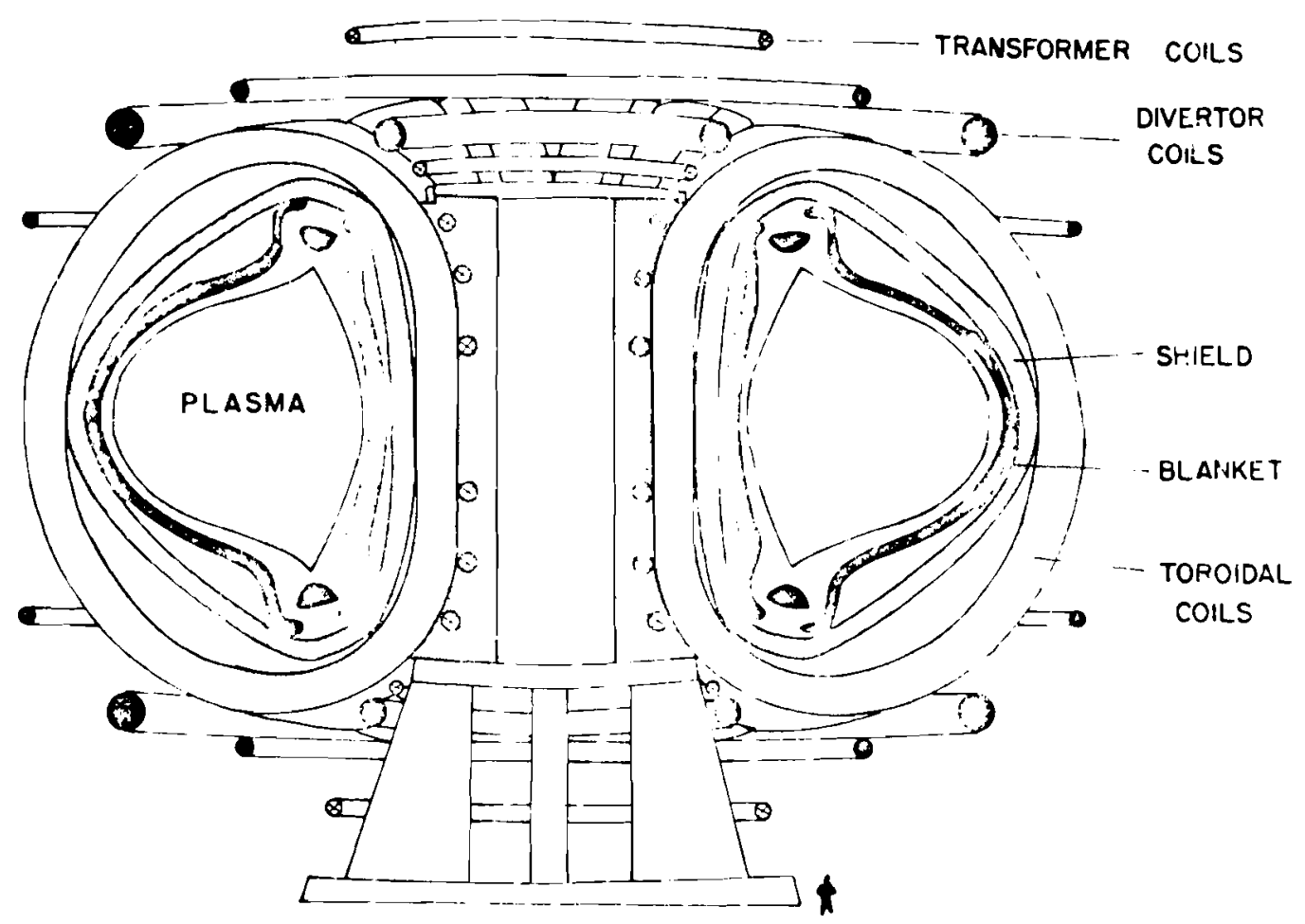

FIGURE 2 Cross Section View of IJWMAK-I Fusion Reactor

Figures 6, 7, and 8 are taken from Yang's calculation. Thic contours of constart toroical field strength on the horizuntal midplane through the torus for the 12 coil case are shown in Figure 6. The toroidal field magnitude from the diagram is less than 0.5 gauss outside the exclusion area (50 meters) at the ground level, and qulckiy drops to near zero at larger distances. Ionizing radiation after fusion reactor startup prohibits workers from routinely entering regions of higher toroidal field strength, although workers might conceivably be exposed to the 10 kilugauss toroidal fields in "tunıng up" a tokamak. jome workers with betatrons, calutrons, magrets for studying Hall effect, crarged particle beam bonding magnets, and other scientific application magnets, have been exposed to such magneiic fields for periojs of an hour or less for several decades with no apparent deleterious effects. 


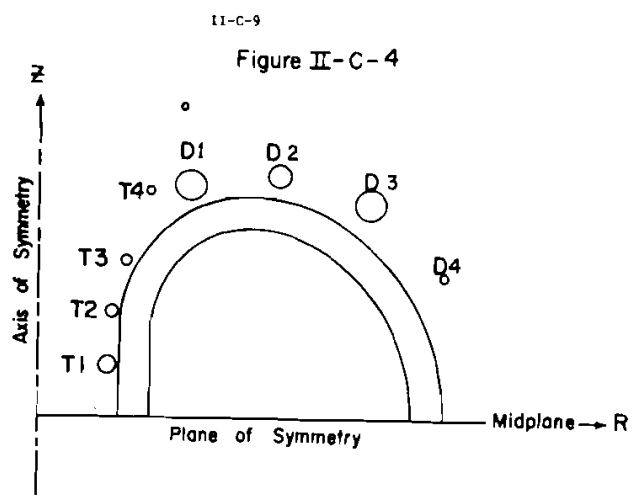

$$
\begin{aligned}
& \text { Coil No. } R(\mathrm{~m}) \quad Z(\mathrm{~m}) \quad I\left(10^{\circ} \mathrm{amp}\right)
\end{aligned}
$$

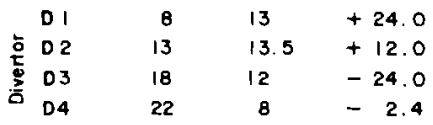

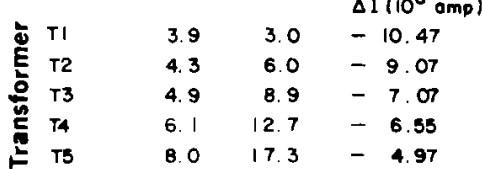

FIGURE 3 UWMAK-I Transformer and Divertor Currents

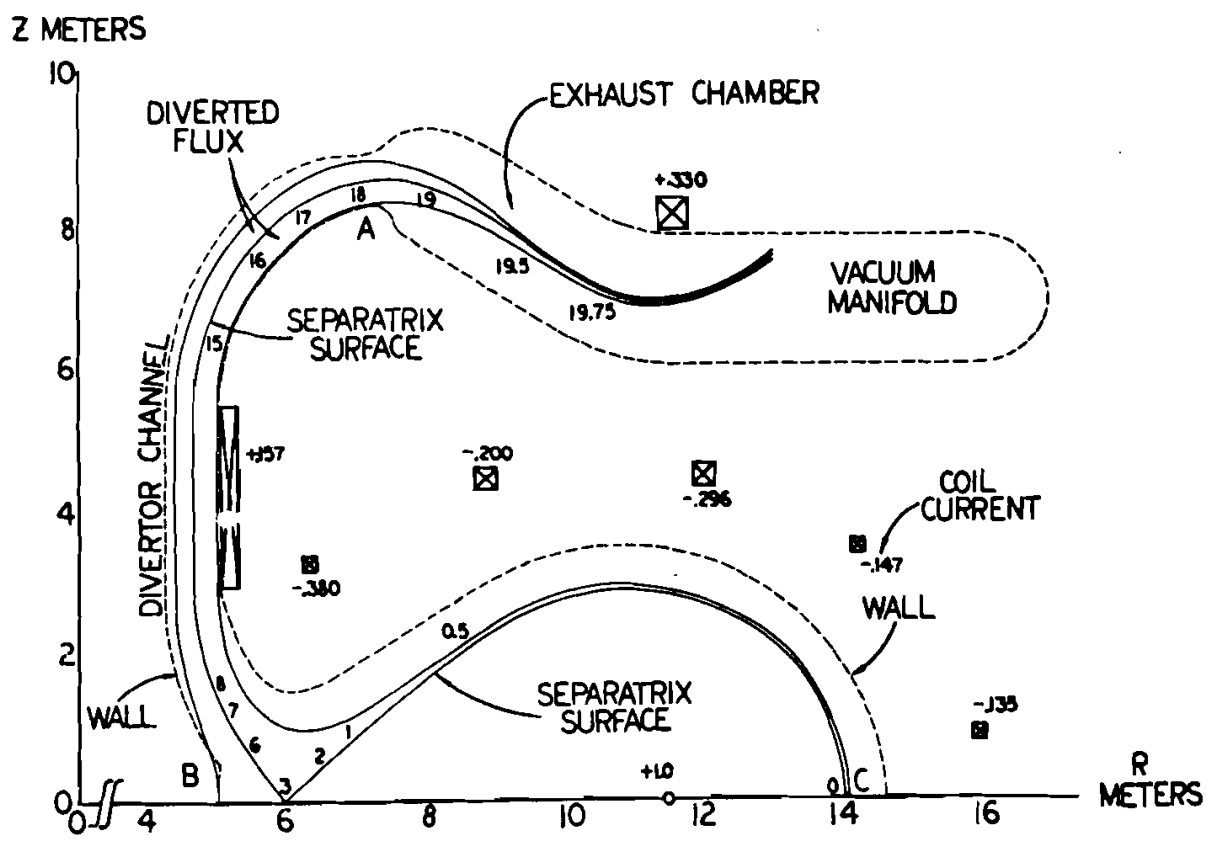

FIGURE 4 Magnetic Flux Surfaces, Divertors and Coils in PPPL Tokamak Design 


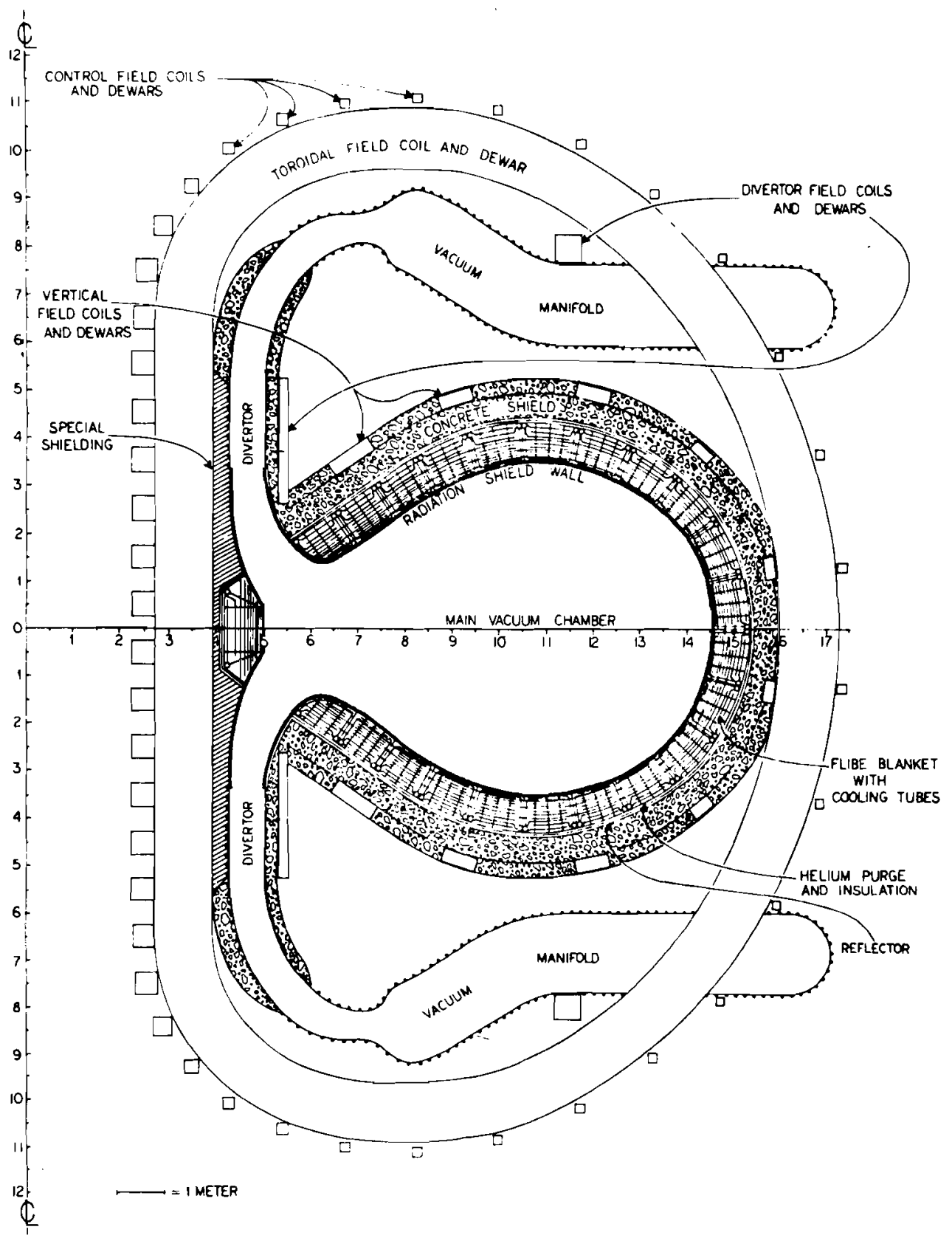

FIGURE 5 Cross Section of the Nuclear Island in PPPL Tokamak Design 


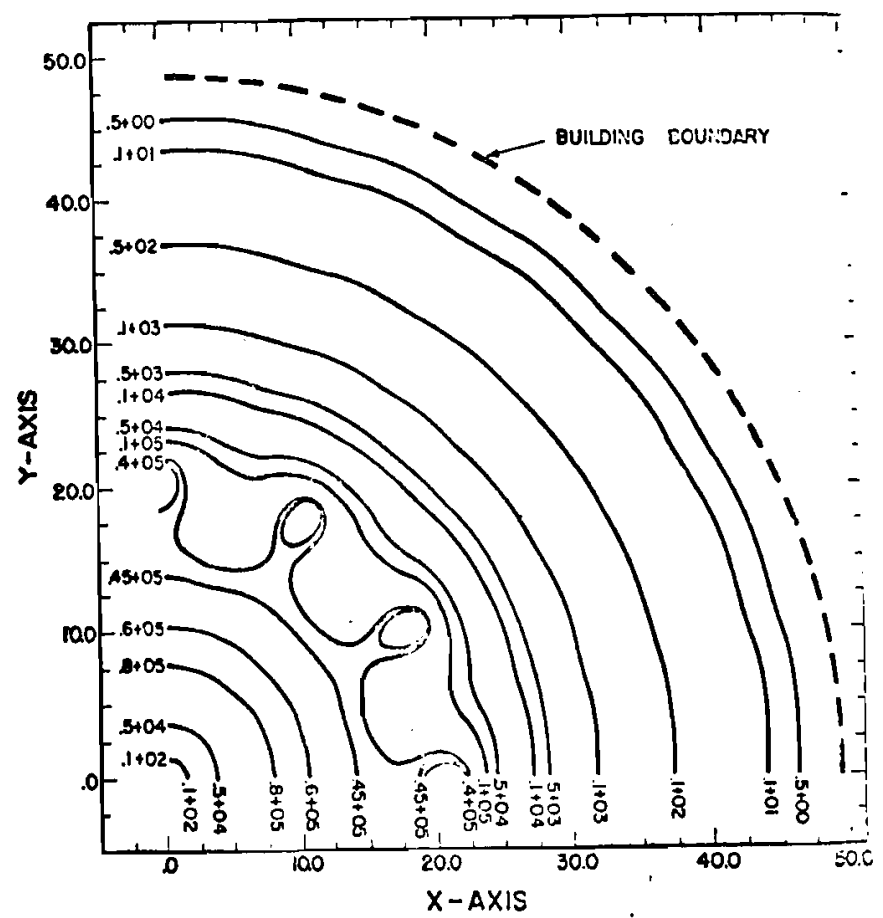

FIGURE 6 Magnetic Field Magnitude Contours in UWMAK-I Midplane. (Magnetic field magnitudes in gauss)

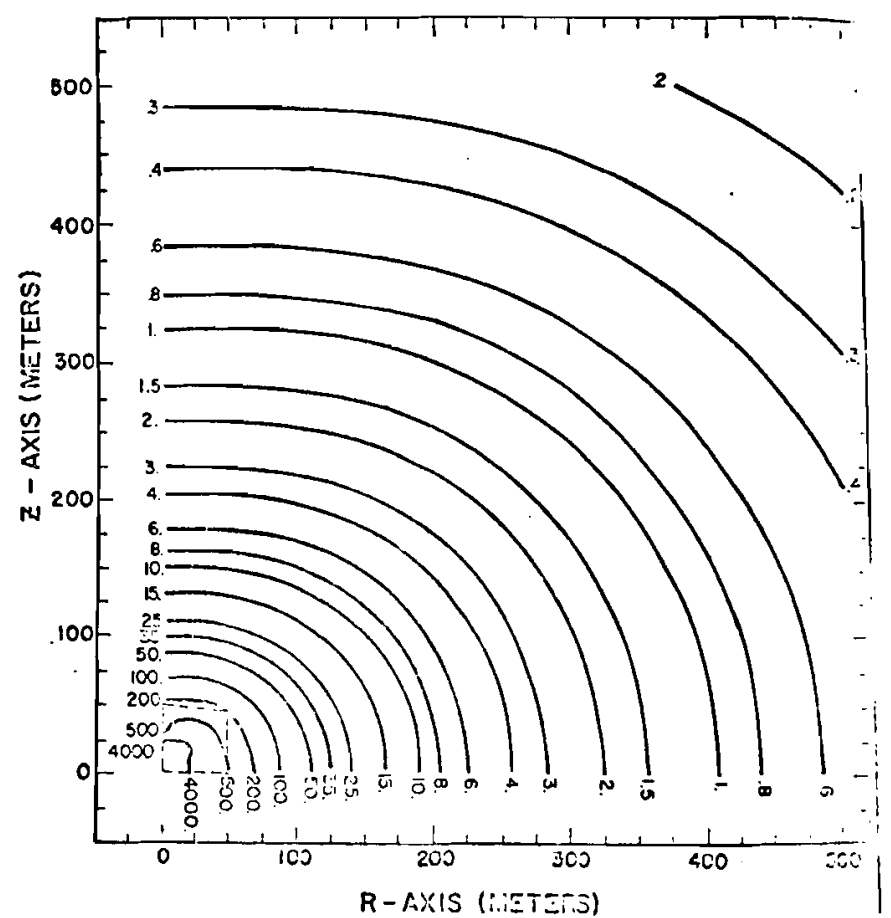

FIGURE 7 Magnetic Field Magnitude Contours in Vertical Plane for UWMAK-I. (Magnetic field magnitudes in gauss) 


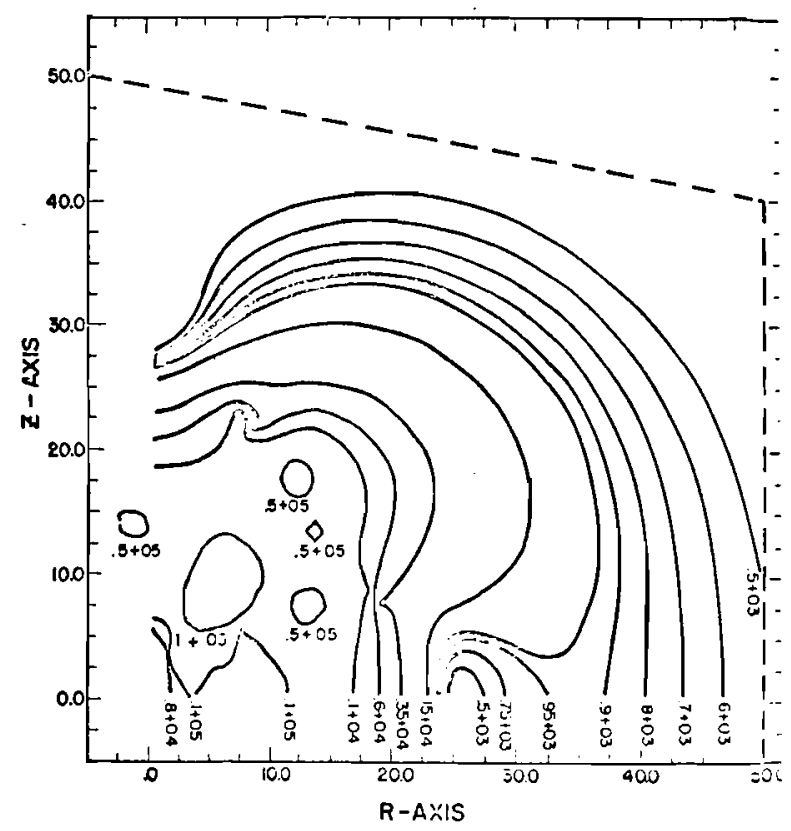

FIGURE 8 Near Magnetic Field Magnitude Contours in Vertical Plane for UWMAK-I. (Magnetic field magnitudes in gauss)

Figure 7 shows the poloidal field contours for the UWMAK-I in the $r-z$ vertical plane with more detail at close distances shown in Figure 8. From Figure 7 we see that a 500 gauss poloidal field would exist outside the exclusion region at midplane, falling to 21 gauss at 400 meters. Field strength above the reactor on torus axis is on the order of half those at the corresponding distance at midplane, that being a characteristic of the dipole field. The field immediately above the building in UWMAK-I is approximately 200 gauss.

Calculational methods generally are adequately developed for determining the locations of magnetic fields around a fusion reactor for simple geometries. However, uncertainties still exist in the effects of complex structures around the reactor on those fields.

As part of the environmental research program, it is advisable to measure the magnitude and configuration of the external magnetic fields around existing large-scale plasma confinement devices. Such measurements would provide an empirical basis for estimating the external fields of future power reactors. Near-field configurations would provide valuable data for the effective multipole structure of the source region. Measurements around reinforced concrete walls and other permeable structures would help define the effect of reactor hall buildings on the magnetic field.

Measurements of the magnetic environment around selected currently operating experiments would also provide a basis for estimating the total magnetic flux exposure of experimenters. From the operations $\log$ it might be possible to reconstruct the gauss-hour exposure of personnel. This medical history would significantly enlarge the statistical data base for investigations of possible biomagnetic effects. 


\section{METHODS FOR REDUCING MAGNETIC FIELDS}

B. H. Duane

Adequately attenuating the leakage of sharply pulsed or rapidly variant magnetic fields, by the use of appropriately cooled copper shielding, appears to lie weil within the state-of-the-art of standard electrical engineering practice, even for the extreme case of abrupt lightning-flash collapse of superconductivity throughout the main confinement coils. Adequately attenuating the leakage of steady-state or slowly variant magnetic fields emerges as the most difficult aspect of designing the magnetic shield for a fusion reactor.

For the LoS Alamos toroidal-pinch reactor design, stray magnetic fields appear to be dominated by the 176th azimuthal harmonic of the pulsed toroidal confinement field. For the Livermore mirror designs, stray magnetic fields appear to be dominated by the dipole component of the pulsed quadrupole confinement field. For the Los Alamos laser-implosion reactor design, stray magnetic fields appear to be dominated by the steady-state dipole divertor field protecting the inner wall. The Livermore laser-implosion work appears to be focused more upon feasibility experiments than upon power reactor design, at present.

The Livermore magnetic bottle has a magnetic moment of $1.1 \times 10^{10}$ Ampere.meter ${ }^{2}$ for the fusionfission hybrid design. (5) The leakage magnetic field falls off inversely as the cube of the distance from the dipole source to values of $2.2 \mathrm{Volt}$.second/meter ${ }^{2}$ along the dipole axis and $1.1 \mathrm{Volt} \cdot$ second/meter ${ }^{2}$ transverse to the dipole axis at a distance of 10 meters.

To reduce stray magnetic fields to an acceptable level, we considered enclosing an entire fusion reactor in a spherical shell of high magnetic permeability material. To bracket available design possibilities, we considered both pure iron (with magnetic permeability 200,000 times that of a vacuum and with a saturation magnetic force field of $2.15 \mathrm{Volt}$. second/meter ${ }^{2}$ ) and Supermalloy (a nickel-iron-molybdenum alloy with magnetic permeability 1,000,000 times that of a vacuum and with a saturation magnetic force field of $0.79 \mathrm{Volt} \cdot$ second/meter $^{2}$ ). (6)

The inner radius of the spherical shell magnetic shield must be sufficiently large to avoid magnetic saturation at its inner surface, and the shell thickness would at most be such as to attenuate the leakage magnetic force field to that of the natural environment (which ranges from $3.12 \times 10^{-5} \mathrm{Volt} \cdot$ second/meter ${ }^{2}$ at the magnetic equator to $6.24 \times 10^{-5} \mathrm{Volt} \cdot$ second/meter $^{2}$ at the magnetic poles). (7) The relevant magnetic attenuation theory is developed in closed form in the following sections.

Shielding the Livermore magnetic dipole moment of $1.1 \times 10^{10}$ Ampere.meter ${ }^{2}$ in accordance with these considerations would require either a pure iron spherical shell 3.1 meters thick with an inner radius of 11.5 meters, or a spherical shell of Supermalloy 0.40 meters thick with an inner radius of 16.1 meters. 
The physical principles for local reduction of magnetic fields are well established and readily applied as necessary. Thus, there is no apparent need for further engineering research in this area at the present time, al though three-dimensional applications are extremely difficult. The design of prototype reactors should include some provision for shielding and local field reduction, however, to ascertain realistic cost estimates for production power reactors.

\section{SPHERICAL MAGNETIC SHIELD ENCLOSING MAGNETIC DIPOLE SOURCE}

To reduce stray magnetic fields to an acceptable level, we considered enclosing an entire fusion reactor in a spherical shell of soft iron, as depicted in Figure 9. The multi-region magnetic field equations relevant to the slowly variant or steady-state magnetic dipole fields most difficult to attenuate take the form: ${ }^{(8)}$

$$
\begin{array}{ll}
\nabla \cdot \underline{B}=0, & \underline{B}=\mu \underline{H}, \\
\nabla \times \underline{H}=0, & B_{\text {normal }} \text { continuous, } \underline{H}_{\text {tangential }} \text { continuous, }
\end{array}
$$

where (in rationalized mks units) $\underline{B}\left(\mathrm{~V} \cdot \mathrm{s} / \mathrm{m}^{<}\right)$is the magnetic force field, $\underline{H}(\mathrm{~A} / \mathrm{m})$ is the magnetic induction field, and $\mu(\mathrm{V} \cdot \mathrm{s} / \mathrm{A} \cdot \mathrm{m})$ is the magnetic permeability.

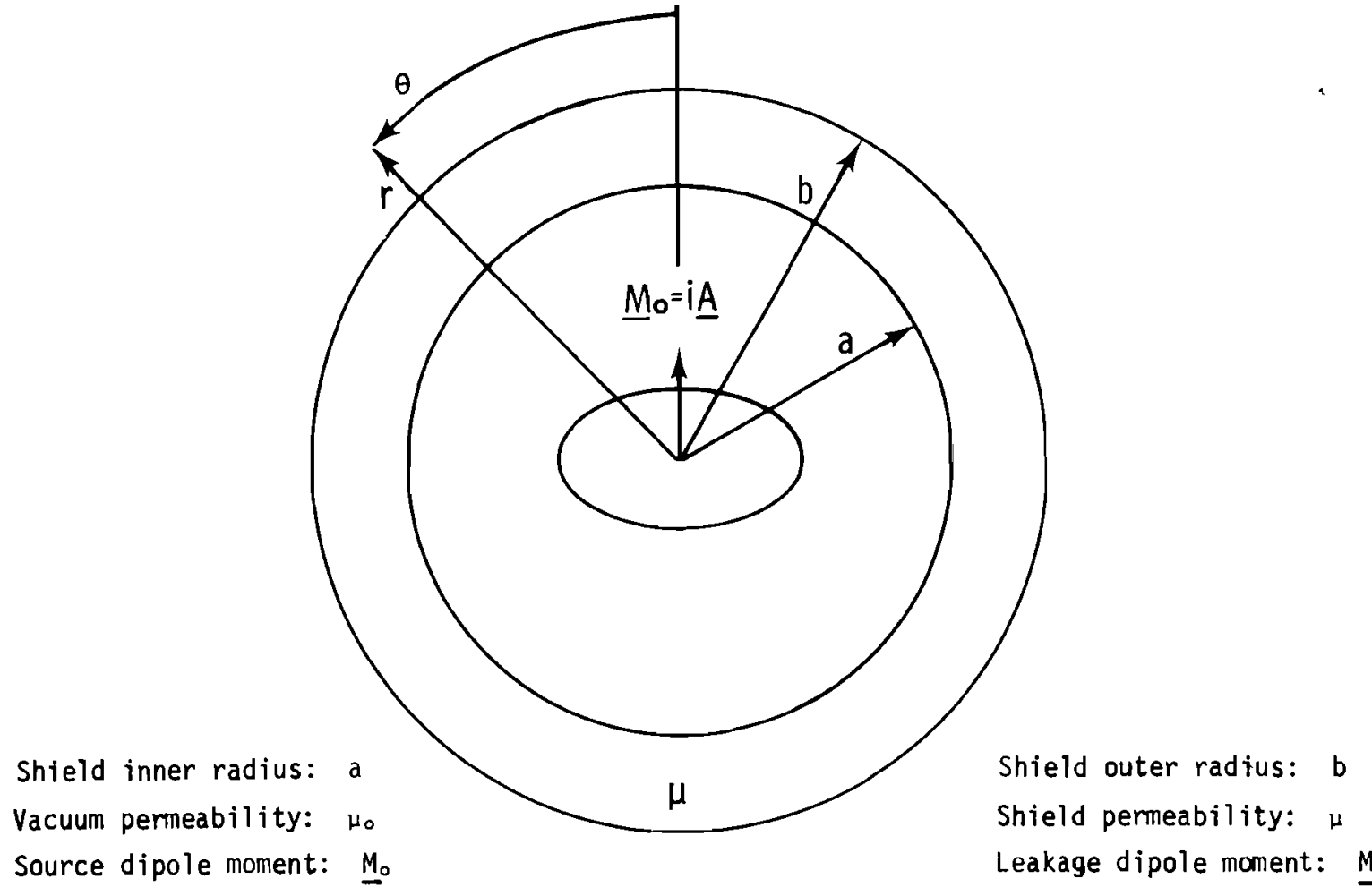

$$
M / M_{0}=\left[1+(4 / 9)\left\{\left(\mu / \mu_{0}+\mu_{0} / \mu\right) / 2-1 ;\left(1-a^{3} / b^{3}\right)\right]^{-1}\right.
$$

FIGURE 9 Spherical Magnetic Shield Enclosing Magnetic Dipole Source 
The magnetic fields can be generated quite simply by beginning with the outermost region and progressing inward through the material interfaces with the help of the identities

$$
\begin{aligned}
& -\nabla\left[(\cos \theta) / r^{2}\right]=\left(2 \underline{u}_{r} \cos \theta+\underline{u}_{\theta} \sin \theta\right) / r^{3}, \\
& \nabla(r \cos \theta)=\underline{u}_{r} \cos \theta-\underline{u}_{\theta} \sin \theta, \\
& 3 \underline{u}_{r} \cos \theta=\left(2 \underline{u}_{r} \cos \theta+\underline{u}_{\theta} \sin \theta\right)+\left(\underline{u}_{r} \cos \theta-\underline{u}_{\theta} \sin \theta\right), \\
& 3 \underline{u}_{\theta} \sin \theta=\left(2 \underline{u}_{r} \cos \theta+\underline{u}_{\theta} \sin \theta\right)-2\left(\underline{u}_{r} \cos \theta-\underline{u}_{\theta} \sin \theta\right),
\end{aligned}
$$

to obtain, sequentially:

$$
\begin{aligned}
& \underline{B}(r>b)=-\nabla\left[\left(\mu_{0} M / 4 \pi r^{2}\right) \cos \theta\right]=\left(\mu_{0} M / 4 \pi r^{3}\right)\left(2 u_{r} \cos \theta+u_{\rightarrow} \sin \theta\right) . \\
& \underline{B}(r=b)=\left(\mu_{0} M / 4 \pi b^{3}\right)\left(2 \underline{u}_{r} \cos \theta+\underline{u}_{-} \sin \theta\right) \text {. } \\
& \underline{B}(a<r<b)=\left(\mu_{0} M / 4 \pi b^{3}\right)\left[(2 / 3)\left\{\left(b^{3} / r^{3}\right)\left(2 \underline{u}_{r} \cos \theta+\underline{u}_{\theta} \sin \theta\right)+\left(\underline{u}_{r} \cos \theta-\underline{u}_{\theta} \sin \theta\right)\right\}\right. \\
& \left.+\left(u / 3 \mu_{0}\right)\left\{\left(b^{3} / r^{3}\right)\left(2 u_{r} \cos \theta+u_{-\theta} \sin \theta\right)-2\left(\underline{u}_{r} \cos \theta-u_{\theta} \sin \theta\right)\right\}\right] \text {. } \\
& \underline{\theta}(r=a)=\left(\mu_{0} M / 4 \pi b^{3}\right)\left[\left\{\left(2+\mu / \mu_{0}\right) / 3\right\}\left(b^{3} / a^{3}\right)\left(2 \underline{u}_{r} \cos \theta+u_{-} \sin \theta\right)\right. \\
& \left.+(2 / 3)\left(1-\mu_{1} / \mu_{0}\right)\left(\underline{u}_{r} \cos \theta-\underline{u}_{\theta} \sin \theta\right)\right] \text {, } \\
& =\left(\mu_{0} M / 4 \pi b^{3}\right)\left[(2 / 3)\left\{\left(2+\mu / \mu_{0}\right)\left(b^{3} / a^{3}\right)+\left(1-\mu_{0} / \mu_{0}\right)\right\}_{u_{r}} \cos \theta\right. \\
& \left.+(1 / 3)\left\{\left(2+\mu / \mu_{0}\right)\left(b^{3} / a^{3}\right)-2\left(1-\mu_{0} / \mu_{0}\right)\right\}_{-} \sin \theta\right] \text {. } \\
& \underline{B}(r<a)=\left(\mu_{0} M / 4 \pi b^{3}\right)\left[(2 / 3)\left\{\left(2+\mu_{0} / \mu_{0}\right)\left(b^{3} / a^{3}\right)+\left(1-\mu_{0} / \mu_{0}\right)\right\}\right. \\
& (1 / 3)\left\{\left(a^{3} / r^{3}\right)\left(2 \underline{u}_{r} \cos \theta+\underline{u}_{\theta} \sin \theta\right)+\left(\underline{u}_{r} \cos \theta-\underline{u}_{\theta} \sin \theta\right)\right\} \\
& +\left(\mu_{o} / 3 \mu\right)\left\{\left(2+\mu_{\mu} / \mu_{o}\right)\left(b^{3} / a^{3}\right)-2\left(1-\mu_{0} / \mu_{o}\right)\right\} \\
& \left.(1 / 3)\left\{\left(a^{3} / r^{3}\right)\left(2 u_{r} \cos \theta+\underline{u}_{\theta} \sin \theta\right)-2\left(\underline{u}_{r} \cos \theta-\underline{u}_{-} \sin \theta\right)\right\}\right] \text {, } \\
& =\left(\mu_{0} M / 4 \pi b^{3}\right)\left[(1 / 9)\left\{\left(2+\mu_{0} / \mu\right)\left(2+\mu^{2} / \mu_{0}\right)\left(b^{3} / a^{3}\right)+2\left(1-\mu_{0} / \mu\right)\left(1-\mu / \mu_{0}\right)\right\}\right. \\
& \left(a^{3} / r^{3}\right)\left(2 \underline{u}_{r} \cos \theta+\underline{u}_{\theta} \sin \theta\right)+(2 / 9)\left(\left(1-\mu_{0} / \mu\right)\left(2+\mu / \mu_{0}\right)\left(b^{3} / a^{3}\right)\right. \\
& \left.\left.+\left(1+2 \mu_{0} / \mu\right)\left(1-\mu_{0} / \mu_{0}\right)\right\}\left(\underline{u}_{r} \cos \theta-\underline{u}_{\theta} \sin \theta\right)\right] \text {. }
\end{aligned}
$$

The source magnetic moment can be read off the singular $\left(1 / r^{3}\right)$ vector harmonic here as

$$
M_{0}=(M / 9)\left[\left(2+\mu_{0} / \mu\right)\left(2+\mu / \mu_{0}\right)+2\left(1-\mu_{0} / \mu\right)\left(1-\mu_{0} / \mu_{0}\right)\left(a^{3} / b^{3}\right)\right] \text {, }
$$

which yields for the leakage/source magnetic moment ratio $M / M_{\circ}$,

$$
M / M_{0}=1 /\left[1+(4 / 9)\left\{\left(\mu / \mu_{0}+\mu_{0} / \mu\right) / 2-1\right\}\left(1-a^{3} / b^{3}\right)\right] \text {, }
$$

as shown in Figure 9. 


\section{MULTIPLE SPHERICAL MAGNETIC SHIELDS ENCLOSING MAGNETIC MULTIPOLE SOURCES}

To reduce stray magnetic fields to an acceptable level, at minimal cost, we considered enclosing an entire fusion reactor in a multi-layered spherical shell of high-permeability materials, as depicted in Figure 10. The inner shell typically might be pure ion (specific permeability $\left.\mu_{\max } / \mu_{0}=200,000\right)$ sufficiently large in inner radius to avoid saturation $\left(B_{\text {sat }}=2.15 \mathrm{~V} \cdot \mathrm{s} / \mathrm{m}^{2}\right) .(6)$ The outer shell might be Supermalloy $\left(.05 \mathrm{Mo}+.79 \mathrm{Ni}+.16 \mathrm{Fe}, \mu_{\max } / \mu_{0}=1,000,000\right)$ sufficiently large in its inner radius to avoid its lower saturation limit $\left(\mathrm{B}_{\mathrm{sat}}=.79 \mathrm{~V} \cdot \mathrm{s} / \mathrm{m}^{2}\right) .(6)$

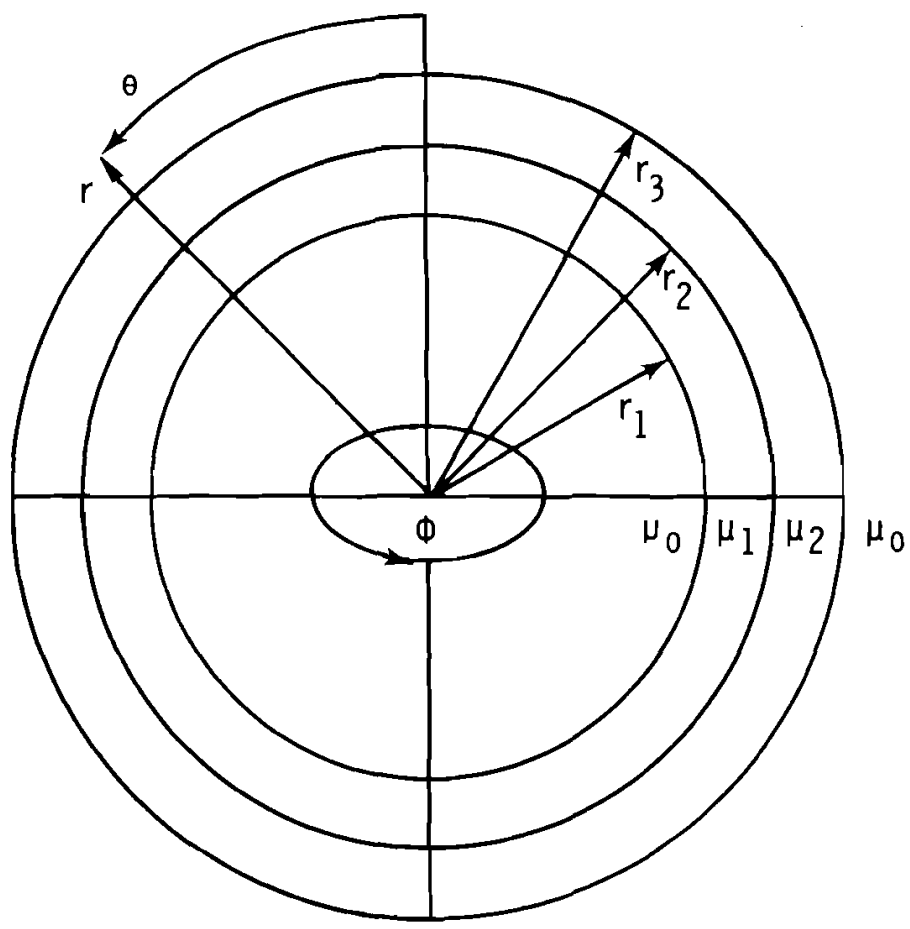

Interface Radii: $r_{n}, n=1, N$

Magnetic Permeabilities: $\mu_{n}, n=0, N$

Source Multipole Moments: $\alpha_{0}^{\ell m}, m=-\ell, \ell ; \ell=1, \infty$

Leakage Hul.tipole Moments: $\alpha_{N}^{\ell m}, m=-\ell, \ell ; \ell=1, \infty$

Reflected Multipole Wells: $\ell_{0} m, m=-\ell, \ell ; \ell=1, \infty$

$$
\left(\begin{array}{l}
\alpha_{0}^{\ell m} / \alpha_{N}^{\ell m} \\
\hline \beta_{0}^{\ell m} / \alpha_{N}^{\ell m}
\end{array}\right)=\prod_{n=1}^{n=N}\left(\begin{array}{l|l}
1+\left(\mu_{n-1} / \mu_{n}-1\right) \ell /(2 \ell+1) & \left(1-\mu_{n-1} / \mu_{n}\right) r_{n}^{2 \ell+1} \ell /(2 \ell+1) \\
\hline\left(1-\mu_{n-1} / \mu_{n}\right) r_{n}^{-2 \ell-?}(\ell+1) /(2 \ell+1) & 1+\left(\mu_{n-1} / \mu_{n}-1\right)(\ell+1) /(2 \ell+1)
\end{array}\right) \cdot\left(\begin{array}{l}
1 \\
- \\
0
\end{array}\right)
$$

FIGURE 10 Concentric Spherical Shell Shields Enclosing Magnetic Multipole Sources 
The multi-region magnetic field equations relevant to the slowly variant or steady-state magnetic fields most difficult to attenuate take the form $(6)$

$$
\begin{aligned}
\nabla \cdot \underline{B}=0, & \underline{B}=\mu \underline{H}, \\
\nabla \times \underline{H}=0, & B_{\text {normal }} \text { continuous, } \underline{H}_{\text {tangential }} \text { continuous, }
\end{aligned}
$$

where (in rationalized mks units) $\underline{B}\left(\mathrm{~V} \cdot \mathrm{s} / \mathrm{m}^{2}\right)$ is the magnetic force field, $\underline{H}(\mathrm{~A} / \mathrm{m})$ is the magnetic induction field, and $\mu(\mathrm{V} \cdot \mathrm{s} / \mathrm{A} \cdot \mathrm{m})$ is the magnetic permeability. The magnetic fields can be generated quite simply by beginning with the outermost region and progressing inward through successive material interfaces with the heip of the identities

$$
\begin{aligned}
& -\nabla\left[r^{-\ell-1} p_{\ell}^{m}(\cos \theta) e^{i m \phi}\right]=r^{-\ell-2}\left[(\ell+1) \underline{u}_{-} P_{\ell}^{m}(\cos \theta)+\underline{u}_{-} \sin \theta P_{\ell}^{m_{1}}(\cos \theta)-i m \underline{u}_{\phi} \csc \theta P_{\ell}^{m}(\cos \theta)\right] e^{i m \phi}, \\
& \nabla\left[r^{\ell} p_{\ell}^{m}(\cos \theta) e^{i m \phi}\right]=r^{\ell-1}\left[\underline{u}_{r} P_{\ell}^{m}(\cos \theta)-\underline{u}_{-} \sin \theta P_{\ell}^{m_{1}}(\cos \theta)+i m \underline{-}_{\phi} \csc \theta P_{\ell}^{m}(\cos \theta)\right] e^{i m \phi},
\end{aligned}
$$

where $P_{\ell}^{m}(\cos \theta) e^{i m \phi}$ spans the complete set of angutar harmonic eigenfunctions. ${ }^{(8)}$

Within a spherical shell having magnetic permeability $\mu_{n}$, the magnetic force field spherical harmonic components $\underline{B}_{l}^{m}$ take the form

$$
B_{l}^{m}\left(r_{n}<r<r_{n+1}\right)=\nabla\left[\left[-a_{n}^{\ell m} \cdot r^{-l-1}+\beta_{n}^{l m} \cdot r^{\ell}\right\} P^{m}(\cos \theta) e^{i m \phi}\right],
$$

with the expansion coeficients $\left(\alpha_{n}^{l m}, B_{n}^{l m}\right)$ determined by the sequential interface continuity for $B_{\text {normal }}$ and $\underline{H}_{\text {tangential }}$ respectively,

$$
\begin{aligned}
& a_{n}^{\ell m}(\ell+1) r_{n+1}^{-\ell-2}+\beta_{n}^{\ell m} \ell r_{n+1}^{\ell-1}=a_{n+1}^{\ell m}(\ell+1) r_{n+1}^{-\ell-2}+\beta_{n+1}^{\ell m} \ell r_{n+1}^{\ell-1} \text {, } \\
& \mu_{n}^{-1}\left(\alpha_{n}^{l m} \cdot r_{n+1}^{-l-2}-\beta_{n}^{l m} \cdot r_{n+1}^{l-1}\right)={ }_{n+1}^{-1}\left(\alpha_{n+1}^{l m} \cdot r_{n+1}^{-l-2}-\beta_{n+1}^{l m} \cdot r_{n+1}^{l-1}\right) \text {. }
\end{aligned}
$$

Progressing inward through an interface by solving for the expansion coefficients on the left yields the matrix interface solution

$$
\left(\begin{array}{c}
\alpha_{n}^{\ell m} \\
\hline \beta_{n}^{\ell m}
\end{array}\right)=\left(\begin{array}{l|l}
1+\left(\mu_{n} / \mu_{n+1}-1\right) \ell /(2 \ell+1) & \left(1-\mu_{n} / \mu_{n+1}\right) r_{n+1}^{2 \ell+1} \ell /(2 \ell+1) \\
\hline\left(1-\mu_{n} / \mu_{n+1}\right) r_{n+1}^{-2 \ell-1}(\ell+1) /(2 \ell+1) & 1+\left(\mu_{n} / \mu_{n+1}-1\right)(\ell+1) /(2 \ell+1)
\end{array}\right) \cdot\left(\begin{array}{l}
\alpha_{n+1}^{\ell m} \\
\hline \beta_{n+1}^{\ell m}
\end{array}\right) .
$$

Cascading inward through all material interfaces by sequential matrix multiplication now generates the complete solution in the explicit form

$$
\left(\begin{array}{l}
\alpha_{0}^{\ell m} \\
\hline \beta_{0}^{\ell m}
\end{array}\right)=\prod_{n=1}^{n=N}\left(\begin{array}{l|l}
1+\left(\mu_{n-1} / \mu_{n}-1\right) \ell /(2 \ell+1) & \left(1-\mu_{n-1} / \mu_{n}\right) r_{n}^{2 \ell+1} \ell /(2 \ell+1) \\
\hline\left(1-\mu_{n-1} / \mu_{n}\right) r_{n}^{-2 \ell-1}(\ell+1) /(2 \ell+1) & 1+\left(\mu_{n-1} / \mu_{n}-1\right)(\ell+1) /(2 \ell+1)
\end{array}\right) \cdot\left(\begin{array}{l}
\alpha_{N}^{\ell m} \\
\frac{\beta_{N}^{\ell m}}{3}
\end{array} .\right.
$$

Both the source/leakage fractions $\alpha_{0}^{l m} / \alpha_{N}^{l m}$ and the reflection/leakage fractions $\beta_{0}^{l m} / \alpha_{N}^{l m}$ can be read off this complete solution, simply by noting that vanishing of the magnetic field at infinite radius implies $\beta_{N}^{l m}=0$, which reduces the preceding equation to 


$$
\left(\begin{array}{l}
\alpha_{0}^{\ell m} / \alpha_{N}^{\ell m} \\
\hline \beta_{0}^{\ell m} / \alpha_{N}^{\ell m}
\end{array}\right\}=\prod_{n=1}^{n=N}\left[\begin{array}{l|l}
1+\left(\mu_{n-1} / \mu_{n}-1\right) \ell /(2 \ell+1) & \left(1-\mu_{n-1} / \mu_{n}\right) r_{n}^{2 \ell+1} \ell /(2 \ell+1) \\
\hline\left(1-\mu_{n-1} / \mu_{n}\right) r_{n}^{-2 \ell-1}(\ell+1) /(2 \ell+1) & 1+\left(\mu_{n-1} / \mu_{n}-1\right)(\ell+1) /(2 \ell+1)
\end{array}\right] \cdot\left[\begin{array}{l}
1 \\
- \\
0
\end{array}\right) .
$$

Tables 3 and 4 convey these ideas to a programmable pocket calculator for numeric evaluation.

TABLE 3 Concentric Spherical Shell Magnetic Multipole Shields (BNW Program Shield)

\section{HP-65 User Instructions}

I the A: SPHERICAL-SHELL DIPOLE SHIELD. B: MULTI-SHELL MULTI-POLE SHIELD P.4. 1 if 2

PH.

SPHER ICAL SHELL MAGNETIC SHIELD

\section{A:1 B:ANY}

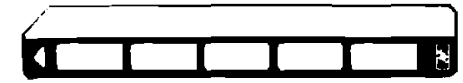

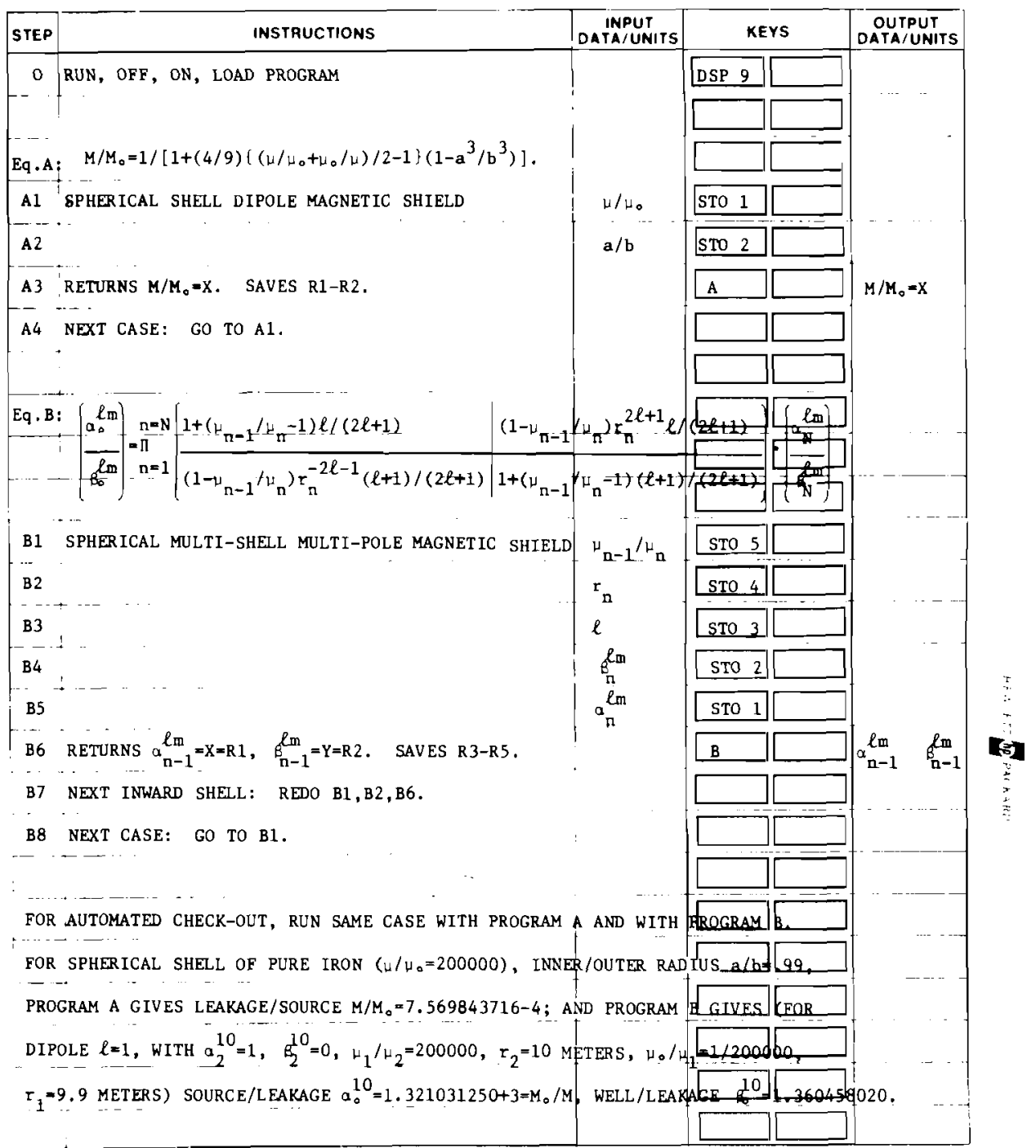


TABLE 4 Concentric Spherical Shell Magnetic Multipole Shields (BNW Program Shield)

\section{HP-65 Program Form}

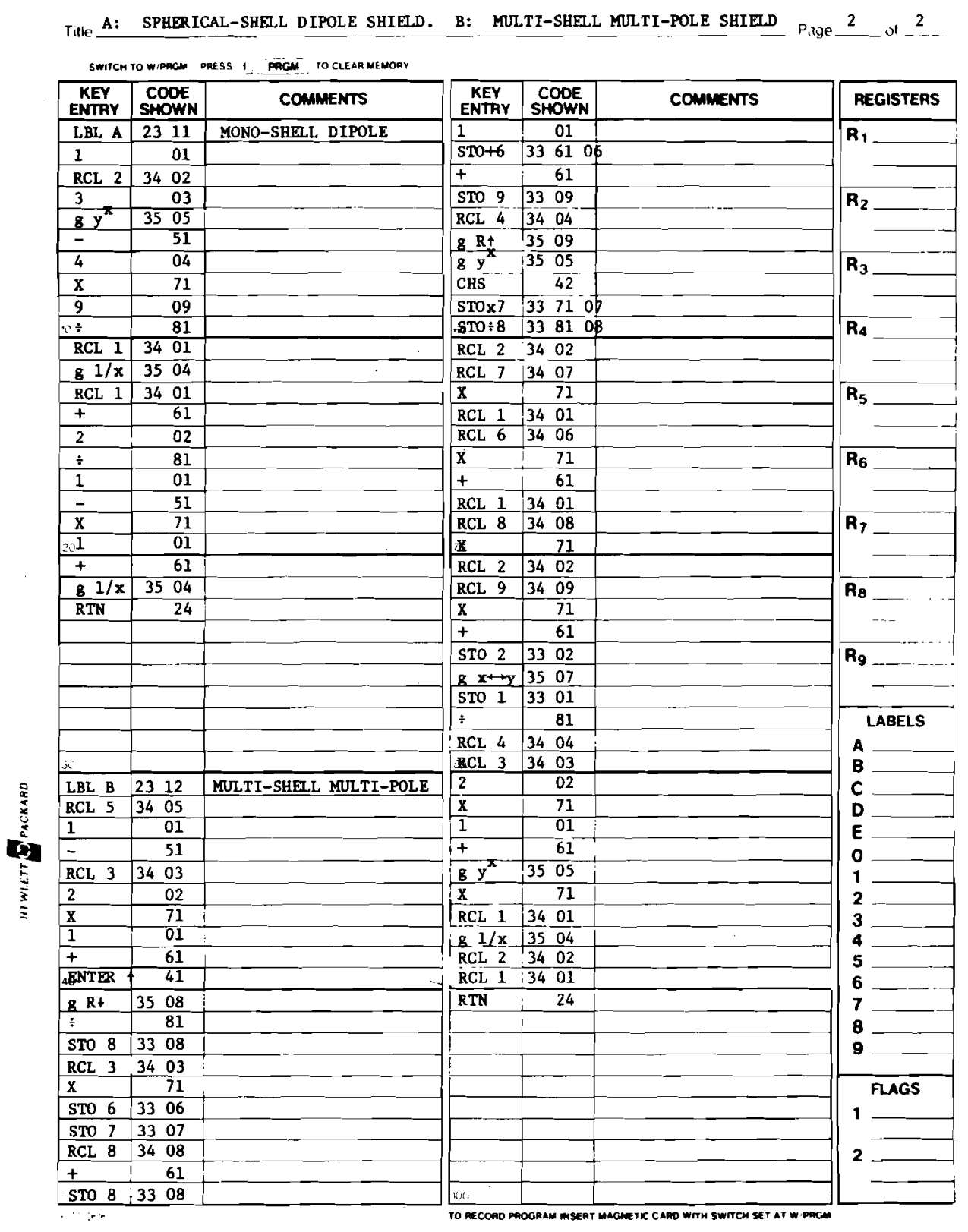




\section{ECONOMICS OF MAGNETIC FIELD REDUCTIONS}

\section{L. Lessor}

The analytical results in Section II provide a good basis for estimating economic factors of magnetic field reduction. The spherical shell model does not provide applicable quantitative results, but it does allow good order of magnitude estimates. There are several materials with high permeability that might be useful for shielding. An important characteristic is the magnetic saturation field that the material is capable of containing. In order to assess economic feasibility, these factors as well as shell location and thickness are varied over a range of probable values for tokamaks.

Consider a spherical shell of magnetic material of outer radius b and inner radius a enclosing a tokamak reactor. The dipole field magnitude $B$ at points outside this shell will be reduced compared with the field $B_{0}$ that would be there without shielding but with the same dipole source by a factor

$$
\frac{B}{B_{0}}=\frac{1}{1+\frac{4}{9}\left(\frac{1}{2}\left(\frac{\mu}{\mu_{0}}+\frac{{ }_{0}}{\mu}\right)-1\right)\left(1-\frac{a^{3}}{b^{3}}\right)}
$$

For highly permeable material $\left(\mu / \mu_{0} \gg 1\right)$ and shell thickness, $s$, small compared with shell radius, this reduction is approximately

$$
\begin{aligned}
\frac{\mathrm{B}}{\mathrm{B}_{0}} & \simeq\left(1+\frac{2}{3} \frac{\mu}{\mu_{0}} \frac{\mathrm{s}}{\mathrm{a}}\right)^{-1} \\
& \simeq \frac{3}{2} \frac{\mu_{0}}{\mu} \frac{\mathrm{a}}{\mathrm{s}} \quad\left(\text { for } \frac{2}{3} \quad \frac{\mu}{\mu_{0}} \frac{\mathrm{s}}{\mathrm{a}}>1\right)
\end{aligned}
$$

Thus the transmitted magnetic field is inversely proportional to the product of shell thickness and shell relative permeability for shells thick enough and permeable enough to give significant field reductions. The shell thickness required for a given reduction factor increases linearly with the transverse dimensions of the region to be enclosed:

$$
\frac{\mu}{\mu_{0}} s \simeq \frac{3}{2} \frac{a}{(B / B)}
$$

for $\mu / \mu_{0} \gg 1$ and $\frac{2}{3} \frac{\mu}{\mu_{0}} \frac{s}{a} \gg 1$ and $s \ll a$. We shall see below that the field strength inside the magnetic material must be below saturation in order to have the large values of $\mu / \mu_{0}$ needed for effective shielding with reasonable thickness.

For a tokamak fusion power device as currently conceived, the inner radius of such a magnetic shielding shell would have to be about 50 meters to allow space for magnets and their maintenance operations and to place the magnetic shield out of the reach of the radiation which could change its magnetic permeability properties. To give outside magnetic field reduction by a factor of $100\left(B / B_{0}=.01\right)$ for such a 50 meter inside radius magnetic shell requires 


$$
\frac{\mu}{\mu_{0}} s \simeq 7500 \text { meters }
$$

Thus the required shell thickness $s$ to give 100 -fold reduction in field outside is

$$
\begin{array}{ll}
s \simeq 1.5 \text { meters } & \text { if } \mu / \mu_{0}=5000, \\
s \simeq .75 \text { meters } & \text { if } \mu / \mu_{0}=10,000, \\
s \simeq .075 \text { meters } & \text { if } \mu / \mu_{0}=100,000, \\
s \simeq .0075 \text { meters } & \text { if } \mu / \mu_{0}=1,000,000 .
\end{array}
$$

Assuming any candidate magnetic shielding material has roughly the same density as purified iron, the masses of the shells needed for enclosures of these thicknesses around a tokamak are:

$$
\begin{aligned}
& m \simeq 3.8 \times 10^{8} \text { kilograms } \simeq 420,000 \text { tons for } s \simeq 1.5 \text { meters } \\
& m \simeq 1.9 \times 10^{8} \text { kilograms } \simeq 210,000 \text { tons for } s \simeq .75 \text { meters } \\
& m \simeq 1.9 \times 10^{7} \text { kilograms } \simeq 20,000 \text { tons for } s \simeq .075 \text { meters } \\
& m \simeq 1.9 \times 10^{6} \text { kilograms } \simeq 2,000 \text { tons for } s \simeq .0075 \text { meters }
\end{aligned}
$$

Limits imposed by magnetic saturation of known materials set a lower limit for required mass on

\begin{tabular}{|c|c|c|c|c|}
\hline Material & Composition & $\begin{array}{c}\text { Low } \\
\text { Field } \\
\text { Relative } \\
\text { Permeability } \\
\end{array}$ & $\begin{array}{c}\text { Maximum Relative } \\
\text { Permeability } \\
\text { (at Magnetic } \\
\text { Field Strength } \\
\text { for which } \\
\mu / \mu_{0} \text { is maximum) }\end{array}$ & $\begin{array}{l}\text { Saturation } \\
\text { Magnetic } \\
\text { Induction } \\
\text { (gauss) } \\
\end{array}$ \\
\hline $\begin{array}{l}\text { Iron } \\
\text { (Commercial) }\end{array}$ & 0.2 impurity & 250 & 9,000 & 21,600 \\
\hline $\begin{array}{l}\text { Iron } \\
\text { (Purified) }\end{array}$ & 0.05 impurity & 10,000 & 200,000 & 21,500 \\
\hline Mumetal & $\begin{array}{l}5 \% \mathrm{Cu}, 2 \% \mathrm{Cr}, \\
77 \% \mathrm{Ni} \text {, balance } \\
\text { Fe \& impurities }\end{array}$ & 20,000 & 100,000 & 6,500 \\
\hline Supermalloy & $\begin{array}{l}5 \% \text { Mo, } 78 \% \mathrm{Ni} \\
\text { balance } \mathrm{Fe} \& \\
\text { impurities }\end{array}$ & 100,000 & $1,000,000$ & 7,900 \\
\hline
\end{tabular}
the order of 300,000 tons, as we shall show. To put these masses into perspective, the UWMAK-I design required approximately $10^{7}$ kilograms $\left(2.4 \times 10^{7}\right.$ bs, about 12,000 tons $)$ of copper and a like amount of stainless steel in the toroidal magnet array. The cost of the magnet materials runs on the order of $\$ 5 / \mathrm{kilogram;}$ shield costs would probably be comparable. Magnetic shield masses must not be much larger than magnet masses if the shielding is not to dominate reactor costs. Table 5 shows relative permeabilities and saturation magnetic induction for candidate magnetic shielding materials.

$\underline{\text { TABLE } 5}$ Properties of Nonpermanent Magnetic Materials ${ }^{(a)}$

(a) Source: American Institute of Physics Handbook, Table $5 g-10^{(6)}$ 
The extremely high relative permeabilities are not achievable in thin sheets of material in strong fields, because magnetic saturation would be exceeded. For example, we could not have $1,000,000$ relative pemeability in a 50 meter inside radius, 0.0075 meter thickness shield, because the field in the shell material would have to be on the order of 5000 kilogauss, far in excess of magnetic saturation of known materials.

The shell thickness for high attenuation of magnetic fields has a lower limit set by saturation magnetic field for the material. The flux which would normally penetrate to distances larger than the shell radius must be "shorted" through the magnetic shielding shell, along with supplementary flux attracted by the high permeability shell. The shell must be thick enough to accommodate this flux while remaining below magnetic saturation. The total flux from a dipole that will pass through the equatorial plane annulus of a high permeability magnetic shell about a dipole is approximately

$$
\Phi=B_{0}(a) 3 \pi a^{2}
$$

where $B_{0}(a)$ is the field which would have been present at distance a from the dipole in midplane with no shield present. This flux is related to the magnetic field $B_{\text {mat }}$ in the high permeability shell by

$$
2 \pi a \leq B_{\text {mat }}=B_{0}(a) 3 \pi a^{2} \text {, }
$$

where $s$ is shell thickness. Thus keeping $B_{\text {mat }}$ below the saturation magnetic field $B_{\text {sat }}$ requires a shell thickness $s$ which satisfies

$$
s>3 / 2\left(B_{0}(a) / B_{\text {sat }}\right) \cdot a \text {. }
$$

For an effective magnetic shield of a spherical region of 50 meter radius in which the field $B_{0}(a)$ that would be present without shielding is 500 gauss (tokamak values), the thickness requirements are:

$$
\begin{aligned}
& s \gtrsim 1.74 \text { meters } \simeq 5.72 \text { feet (soft iron) } \\
& s \gtrsim 4.75 \text { meters } \simeq 15.6 \text { feet (supermalloy). }
\end{aligned}
$$

The mass of iron required is thus approximately $4.4 \times 10^{8}$ kilograms or 490 thousand tans, a prohibitive amount. The mass needed for lower saturation magnetic materials is larger still.

The volume of shielding material required in such a magnetic shell does not vary appreciably with inner radius of the shell in the range of radij in which the dipole field predominates. Noting that the midplane unperturbed dipole field varies according to

$$
B_{0}(a)=\left(\mu_{0} / 4 \pi\right) M / a^{3} \text {, }
$$

where $M$ is a measure of the dipole strength $(M \simeq \Sigma I A)$, then the volume $V$ of shielding required is 


$$
V=4 \pi a^{2} s \geq 4 \pi a^{2} \cdot 3 / 2\left(\mu_{0} / 4 \pi\right)\left(M / a^{3}\right)\left(a / B_{s a t}\right)
$$

or

$$
v \gtrsim(3 / 2) \mu_{0} M / B_{\text {sat }} \text {, }
$$

which is independent of the radius a.

There remains a question as to whether any other configuration of shielding material could shield the reactor magnetic fields. It seems unlikely that any magnetic material configuration could contain the tokamak fields with reasonable mass, but it may be possible to provide low field regions for operators if it is deemed necessary to have them located close to the facility. Bucking coils and/or magnetic shields could provide these low field regions.

The thickness needed to give high attenuation shielding for operators inside a spherical magnetic material shell is also determined by magnetic saturation limits. A first approximation to the thickness $s$ needed is given by

$$
s \succsim(3 / 2)\left(B_{0} / B_{\text {sat }}\right) a
$$

where $B_{0}$ is the ambient magnetic field without the shell, $B_{\text {sat }}$ is saturation magnetic field in the shell material, and $a$ is the radius of the region to be shielded. Shielding a 3 meter radius region with iron requires somewhat more than 0.1 meter ( $\sim 4$ inches) of shell thickness at a location where $B_{0}$ is 500 gauss. The thicknesses required are not out of the question for small regions.

At greater distances from the reactor, the shielding thickness needed drops off. The thickness $S$ of shielding required to shield a spherical enclosure of radius a will be

$$
s \gtrsim \operatorname{Max}\left[\frac{3}{2} \frac{\mathrm{B}_{0}}{\mathrm{~B}_{\text {sat }}} \mathrm{a}, \frac{3}{2} \frac{{ }_{0} \mathrm{~B}_{0}}{\mu \mathrm{B}_{\text {perm }}} \mathrm{a}\right]
$$

where $B_{\text {sat }}$ is the saturation magnetic field in the shield material, $B_{0}$ is the ambient magnetic field at the location in the absence of the shield, $B_{\text {perm }}$ is the maximum permissible field inside the enclosure, and $\mu / \mu_{0}$ is the relative permeability of the magnetic shield material. Table 6 shows the thickness, volume, mass, and cost of material (at \$2/pound) to shield the interior of a spherical shell of inner radius 3 meters. Shielding down to a permissible field $B_{\text {perm }}$ of 1 gauss with magnetic material of relative permeability 200,000 and magnetic saturation 21,500 gauss (approximately that of pure iron) is assumed.

The magnetic field present without shielding is assumed to vary as $r^{-3}$ in the manner of a dipole, and values typical of a tokamak power plant are used. The thickness required for the saturation limit is seen to dominate over that required for attenuation to 1 gauss in the range shown. The nonconstancy of relative permeability makes the table only qualitatively correct, but the trend toward less cost to shield smaller fields is obvious. The linear expressions for the thicknesses needed given in the previous paragraph were used. Caution should be used in estimating thicknesses of shielding needed in other geometries, owing to the nature of the magnetic field and the boundary conditions which magnetic field components satisfy at interfaces. 
$\underline{\text { TABLE } 6}$ Typical Operating Area Magnetic Shielding - Tokamak Reactors

\begin{tabular}{|c|c|c|c|c|c|c|}
\hline \multirow[b]{3}{*}{$\begin{array}{l}\text { Distance from } \\
\text { Reactor Center } \\
\text { (meters) } \\
\end{array}$} & \multirow{3}{*}{$\begin{array}{l}\text { Magnetic Field } \\
\text { Strength Wi thout } \\
\text { Shielding } \\
\text { (Gauss) } \\
\end{array}$} & \multicolumn{2}{|c|}{$\begin{array}{c}\text { Shell Thickness } \\
\text { Lower Limits }\end{array}$} & \multirow{2}{*}{\multicolumn{3}{|c|}{ She11 Requirements }} \\
\hline & & \multirow{2}{*}{$\begin{array}{l}\text { Imposed by } \\
\text { Saturation } \\
\text { (meters) }\end{array}$} & \multirow{2}{*}{$\begin{array}{l}\text { Imposed by } \\
\text { Field } \\
\text { Reduction } \\
\text { (meters) } \\
\end{array}$} & & & \\
\hline & & & & $\begin{array}{l}\text { Volume } \\
\text { (meter }{ }^{3} \text { ) }\end{array}$ & $\begin{array}{r}\text { Mass } \\
(\mathrm{kg})\end{array}$ & $\begin{array}{l}\text { Cost } \\
(\$ \text { at } \\
\$ 2 / 1 \mathrm{~b})\end{array}$ \\
\hline 50 & 550 & 0.12 & 0.012 & 14 & 110,000 & 480,000 \\
\hline 100 & 70 & 0.014 & 0.0015 & 1.6 & 12,000 & 54,000 \\
\hline 150 & 20 & 0.0042 & 0.00046 & 0.47 & 3700 & 16,000 \\
\hline 200 & 8.6 & 0.0018 & 0.00019 & 0.20 & 1,600 & 7,000 \\
\hline 300 & 2.5 & 0.00053 & 0.000057 & 0.06 & 470. & 2,000 \\
\hline 325 & 2. & 0.00042 & 0.000045 & 0.05 & 370 . & 1,600 \\
\hline
\end{tabular}

Basis: Spherical shel1, 3 meter inner radius, 1 gauss inner magnetic field, shell relative permeability of 200,000 , magnetic saturation of 21,500 gauss. 


\section{FORCES ON MAGNETIZABLE OBJECTS NEAR MAGNETIC CONFINEMENT FUSION REACTORS}

\section{L. Lessor}

Magnetizable objects experience forces in inhomogeneous magnetic fields. This presents some hazard to persons using or working around iron or steel tools or other magnetizable objects near intense magnetic field sources. The hazard is that of flying projectiles. We shall here estimate size of forces and the extent of the hazardous region around magnetic confinement fusion reactors.

The force on a magnetizable object in a magnetic field whose current sources are fixed is given by ${ }^{(9)}$

$$
F_{i}=\frac{\partial}{\partial X_{i}} w
$$

where $F_{i}$ is the ith component of the generalized force and $X_{j}$ is the $i$ th coordinate of the object position. Here $W$ is the free energy associated with the object in its location. We can take $W$ as the magnetic field energy with the object present minus magnetic field energy with the object absent:

$$
W=\frac{1}{8 \pi} \int\left(\underline{B} \cdot \underline{H}-\underline{B}_{0} \cdot \underline{H}_{0}\right) d^{3} X .
$$

Here $\underline{B}$ and $\underline{H}$ are magnetic force field and magnetic field intensity, respectively, and we assume cgs Gaussian units. For fixed current sources, we can simplify Equation 2 to

$$
W=\frac{1}{8 \pi} \int_{\text {Al1 space }}\left(\underline{B} \cdot \underline{H}_{-0}-\underline{H} \cdot \underline{B}_{-0}\right) d^{3} x
$$

or, assuming a relative permeability $\mu$ in the introduced object and $\mu_{0}$ in the surrounding medium, to

$$
W=\frac{1}{8 \pi}\left(\mu-\mu_{0}\right) \int_{\text {Interior }} \underline{H} \cdot H_{0} d^{3} x,
$$

We can evaluate the free energy $W$ using Equation 4 for objects whose shape permits finding $\underline{H}$ explicitly for a given $H_{0}$. One can then find a free copy per unit volume $W_{v}$ and a corresponding force per unit volume

$$
\underline{F}_{v}=\nabla W_{v} .
$$

Shapes for which $W_{v}$ can be calculated include:

Magnetizable sphere

$$
W_{v}=\frac{1}{8 \pi}\left(\mu-\mu_{0}\right)\left(H_{0}\right)^{2} \frac{3 \mu_{0}}{\mu+2 \mu_{0}}
$$


Magnetizable cylinder with axis perpendicular to magnetic field

$$
W_{v}=\frac{1}{8 \pi}\left(\mu-\mu_{0}\right)\left(H_{0}\right)^{2} \frac{2 \mu_{0}}{\mu+\mu_{0}}
$$

Magnetizable rod with long direction parallel to magnetic field

$$
W_{v}=\frac{1}{8 \pi}\left(\mu-\mu_{0}\right)\left(H_{0}\right)^{2}
$$

Sheet of magnetizable material with surface plane perpendicular to field

$$
W_{v}=\frac{1}{8 \pi}\left(\mu-\mu_{0}\right)\left(H_{0}\right)^{2} \frac{\mu_{0}}{\mu}
$$

Magnetic forces on objects can be large when $\mu$ is much greater than one and $\mu_{0}$ is near one as it is for air, vacuum, and nonmagnetic materials. Equations 6-9 and Equation 5 show that long magnetizable objects with long direction parallel to the magnetic field will experience the largest force per unit volume in a nonuniform magnetic field. Unfortunately, this is the shape of steel tools, iron bolts, saw blades, files, and many other objects likely to be used in and around magnetic confinement fusion reactors.

Workers are accustomed to having tools subject to gravitational forces in use and when dropped. Presumably a magnetic force of comparable magnitude would be tolerable and present no unusual hazards, al though it may require some adjustment to having dropped tools not fall vertically downward. In regions where the magnetic force exceeds typical friction coefficient times gravitational force, some inconvenience from sliding or bounding tools will be encountered. A greater inconvenience, of course, will come from the interaction between tools, between wrench and bolt, etc.

For a long tool or object experiencing magnetic force per unit volume

$$
\underline{F}_{-m}=\frac{1}{8 \pi}(\mu-1) \nabla\left(\underline{H}_{0}^{2}\right)
$$

and gravitational force per unit volume

$$
\underline{F}_{G}=\rho g \text {, }
$$

the ratio of their magnitudes will be

$$
\frac{F_{m}}{F_{G}}=\frac{1}{8 \pi} \frac{(\mu-1)\left|\nabla\left(\underline{H}_{0}^{2}\right)\right|}{\rho g}
$$

We have neglected terms in the gradient of $\mu$. Here $\rho$ is the mass density of the object, $g$ is the acceleration of gravity, and $\underline{H}_{0}$ is the magnetic induction at the position before the object is introduced.

The magnetic field from a tokamak at locations somewhat more distant from axis-midplane intercept than the toroidal field coils will be dominated by the dipole field from poloidal field magnets. The field strength of the UMMAK-I poloidal field is roughly 500 gauss at a point in midplane $50 \mathrm{~m}$ from the symmetry axis. Modification of the vertical and control field coil 
configuration will cause some variation, probably downward, in the distant poloidal magnetic field, but we can use a dipole field of this strength to illustrate order of magnitude of the magnetic force.

The relative permeability of magnetic materials is an intricately varying function of $\mathrm{H}$ for ferromagnetic materials. ${ }^{(6)}$ To use Equation 12 to estimate ratio of magnetic to gravitational forces, we use an approximate empirical form for iron above magnetic saturation:

$$
\begin{aligned}
& \mu=\mathrm{aH}^{\gamma}+1 . \\
& \mathrm{a}=12454 . \\
& \gamma=-.90504
\end{aligned}
$$

This form is accurate to within about $10 \%$ in the range

8. oersteds $<H<1000$ oersteds

and is probably fair for some $\mathrm{H}$ range greater than 1000 oersteds, It should be noted that for a long, thin object with long axis parallel to magnetic induction field, the interior value of $\mathrm{H}$ is equal to the value $H_{0}$ that would be present were the object absent. Equation 13 gives approximately $40 \%$ error at $H=1.5$ oersteds and should not be used for any $\mathrm{H}$ values lower than this.

Figure 11 shows the ratio of magnetic force to gravitational force on long slender iron objects plotted as a function of distance from a magnetic dipole. The dipole strength was set by requiring a magnetic induction field of 500 oersteds (numerically 500 gauss) at a distance of $50 \mathrm{~m}$ in midplane. This is representative of UWMAK-I.

Figure 12 shows contours of constant ratio of magnetic force to gravitational in a plane through the axis of this field. The magnetic force on long thin iron objects is equal to gravitational at about $25 \mathrm{~m}$ distance and on the order of $10 \%$ of gravitational at $50 \mathrm{~m}$.

The hazard from flying objects propelled by magnetic force appears largely confined to the interior of the reactor building. The annoyance to workmen from magnetized tools extends into the exterior beyond $50 \mathrm{~m}$, however It will. probably be necessary to equip workmen with mostly nonferromagnetic tools for work in and around the reactor building. Aluminum and nonmagnetic stainless steels can be used extensively for this purpose.

Sand and soil in many areas contain magnetite and possibly other magnetic materials, as can be seen by running a small magnet over the ground surface. Since loose soil surfaces are likely to be $50 \mathrm{~m}$ or more from the reactor center, magnetic field-induced migration of these materials should not be a problem. Their presence might require some fine tuning of control field coils, however.

From Figure 12, one notes the slow falloff of magnetic forces beyond about $45 \mathrm{~m}$ in this dipole field. This comes from the increase in relative permeability $\mu$ as the magnetic induction drops back from a value sufficient to give near-saturation. The highest value of $\mu$ for magnetic iron occurs at $H$ approximately 1.5 oersteds, at which $\mu$ is approximately 6000 . The value $H_{0}=$ 1.5 oersteds occurs in the UWMAK-I midplane at a distance on the order of $350 \mathrm{~m}$. 


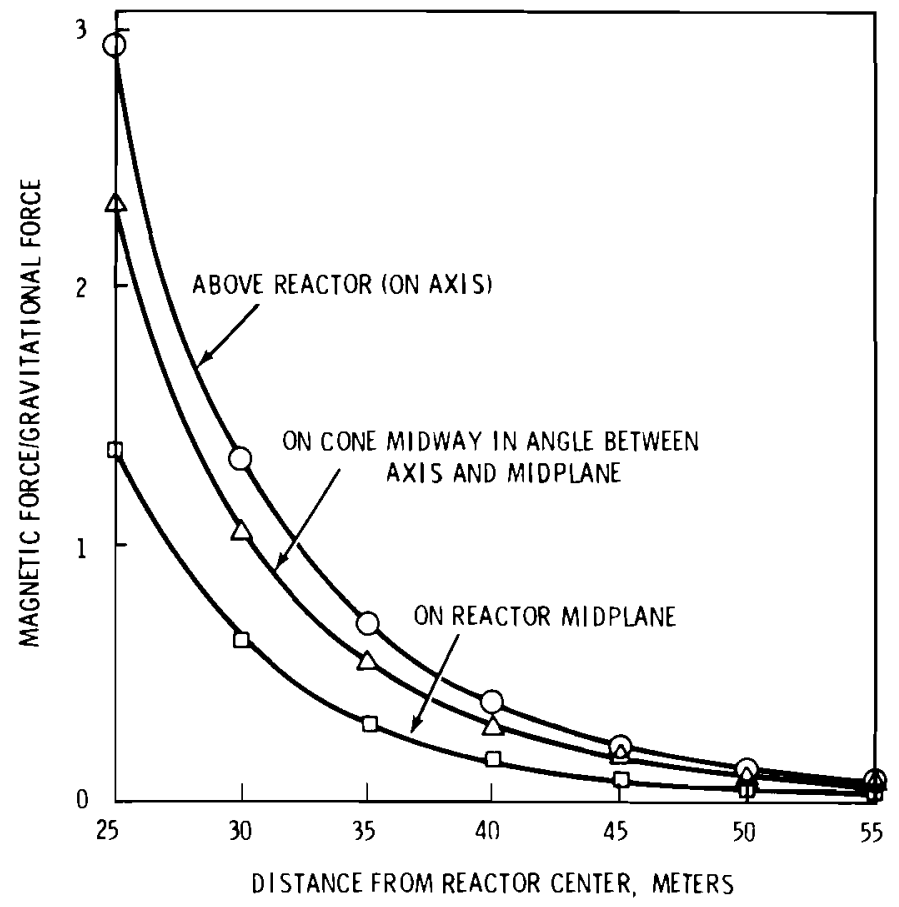

FIGLIRE 11 Ratio of Magnetic Force to Gravitational Force on a Long, Slender, Magnetic Iron object in a Dipole Field, Similar to that of UWMAK-I

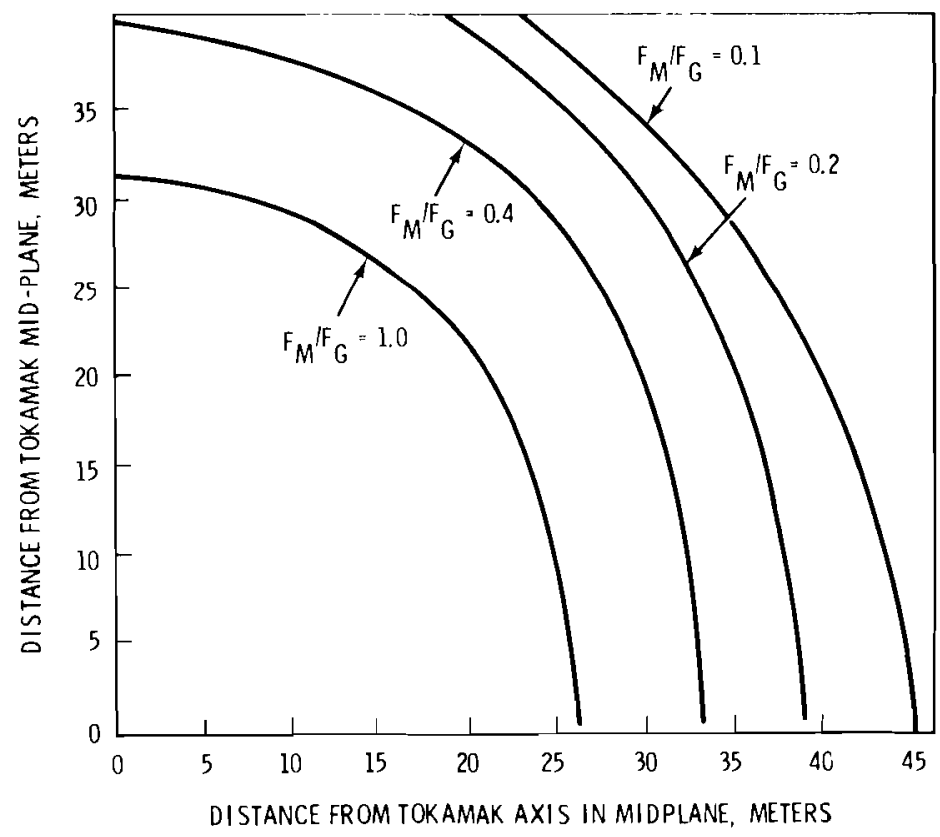

FIGURE 12 Contours of Constant Ratio of Magnetic Force $F_{M}$ to Gravitational Force $F_{G}$ on Long Iron Tools in a Dipole Field Similar to that of UWMAK-I 
One should expect the annoying interaction between magnetizable objects to persist to these distances, even though the force on an isolated object becomes negligible. One can envision situations where this interaction presents safety hazards, as, for example, when magnetic interaction with some other object pulls a workman's hand held tool into contact with electrical leads. The development of a complete line of nonmagnetic tools would seem to solve this problem, and be quite practical. 


\title{
VI. ELECTRIC FIELD TRANSIENTS IN TOKAMAKS
}

\author{
D. L. Lessor
}

The magnetic field $\underline{B}$ in normal operation of a conceptual tokamak fusion power plant is systematically varied for plasma heating and control. The magnetic field change rate $\underline{B}$ results in an induced electrical field $\underline{E}$ at points in space in the tokamak environs. The induced electric field for tokamaks as currently conceived is sufficiently low that hazards from its electric field are at a low level and of a routinely encountered variety. The highest values of $\underline{\mathbf{B}}$ in normal fusion power tokamak operation are associated with poloidal fields during ignition and shutdown.

The toroidal magnetic field generated by currents in the toroidal field coils is brought up to operating value slowly in reasonable tokamak designs, as determined by the tradeoff on cost per unit power for energizing the coils and cost of time delay in bringing up to power an expensive piece of capital equipment. The energizing of toroidal field coils will probably occur directly from utility grid lines rather than from capacitive or inductive storage and will take many minutes to a few hours. The induced electric field will be low compared with those from the poloidal field ignition and shutdown transients, because of the slow magnetic field buildup. The most rapid magnetic field transient is that associated with ignition or shutdown, and the magnetic fields varied are poloidal fields from transformer currents, plasma current, divertor current, and control field current. The procedure during ignition is an intricate one requiring controlled changes in not only the currents but also the plasma density. Low initial pressure inside the torus is favored for startup because joule heating power in the plasma must exceed bremsstrahlung and line radiation power if the temperature is to increase (unless energetic neutral beam injection is supplying sufficient heating), and the radiated power in bremsstrahlung and line radiation is proportional to plasma ion density squared at a given temperature. One would like to achieve the ohmic heating in the shortest practical time consistent with retaining plasma control and keeping cost of the power system reasonable, since rapid heating reduces radiated energy loss. It is particularly important to break quickly out of the low temperature domain below temperatures of $100 \mathrm{eV}$ where line radiation losses are severe. The cost per unit power for energizing the poloidal field coils and for neutral beam plasma heating gives a tradeoff which assures that the ignition period will not be made arbitrarily short, however. Shortening the rise time of magnetic fields also increases the cryogenic refrigeration power needed.

The emf to build up plasma current in startup or restart in a tokamak device is supplied by poloidal field coils acting as a transformer primary. The plasma current buildup obeys approximately a differential equation

$$
-L \frac{d I_{p}}{d t}=I_{p} R+\dot{\Phi}_{\text {ext }}
$$


where $L$ is inductance of the plasma ring carrying toroidal current $I_{p}$ and having electrical resistance $R$. The rate of buildup $\mathrm{dI}_{\mathrm{p}} / \mathrm{dt}$ is determined by the rate of change of external magnetic flux $\Phi_{\text {ext }}$ through the plasma current ring and the other parameters. The plasma inductance $L$ and resistance $R$ are not constant during buildup, being functions of plasma temperature and configuration. There exists some uncertainty (as much as a factor of 10) in plasma resistivity because of trapped electron and impurity effects. The plasma current loop is primarily an inductive rather than resistive load during the ignition period from the time the plasma temperature exceeds 500 electron volts until the time when plasma current is leveled off near its burn phase value by control of $\dot{\Phi}_{\text {ext }}$. Hence the rise time is not very sensitive to plasma resistivity.

The PPPL tokamak design ${ }^{(4)}$ calls for a rise to full plasma current $I_{p}=14.6$ megamperes in 13.25 seconds with $\Phi$ ext $=200$ volts initially, with lower $\Phi_{\text {ext }}$ after departure from the line radiation regime in less than 0.1 second. The electric field $E$ at the center of the minor plasma cross section will be

$$
E=\Phi_{\text {ext }} / 2 \pi R
$$

where $\mathrm{R}$ is the major toroid radius. The electric field at the plasma position in the PPPL design with major radius $R \cong 11.5$ meters would be $E \simeq 2.8$ volts/meter. The electric field at points accessible to workers will be weaker. The electric field induced by the poloidal field coils requires special situations to be hazardous, as, for example, a wire which circles the reactor one or more times and forms a closed circuit when both ends are contacted by a worker, who might then experience a voltage drop in his body of 200 volts times number of turns around the reactor. It is only because of the low pressure in the plasma region that such a low electric field can induce breakdown and cause a buildup of plasma current.

The UWMAK-I design ${ }^{(3)}$ called for a 100 second plasma current buildup, but a subsequent University of Wisconsin design leans toward a 10 second current transient in ignition. The initial design chose 100 second current buildup to keep magnetic field buildup rate $B$ to less than 0.6 kilogauss/ second (or 0.06 tesla/second). A uniform magnetic field buildup at this rate on the interior of a ring of 12 meters radius results in an emf of only 27 volts around its circumference, or an electric field of only 0.36 volts/meter. Building to the same final field in a factor of 10 less time gives corresponding emf of 270 volts and electric field of 3.6 volts/meter, on the same order as those in the PPPL design.

The emf induced in a circular loop encircling the torus at midplane level falls off approximately as $R^{-1}$ with increasing distance $R$ from the torus center, and hence the electric field falls off as $R^{-2}$. The potentials and the electric field will be smaller at the location of the operating personnel than the mentioned values which are typical of those near the torus. To see the falloff with increasing radius of the emf induced in a loop encircling the torus at midplane, note that increasing the radius $R$ increases the amount of return flux through the loop from the magnetic dipole, and hence reduces net flux through the loop and reduces the magnitude of $\dot{\Phi}$ from its variation. 
The normal shutdown transient for a tokamak is of comparable duration to startup in the PPPL ( 20 seconds) and University of Wisconsin designs ( 100 seconds in UWMAK-I). This is because many of the shutdown problems are inverses of startup problems. One must cool the plasma down while retaining control, then store or dissipate the magnetic energy associated with poloidal fields.

Magnetic field transient rates in the two power tokamak designs were determined to a significant extent by the assumption of an upper limit on magnet energizing power of 500 megawatts (PPPL) or 1000 megawatts (UWMAK-I), largely from economic considerations. A tokamak power plant must be large for economy. Hence the amount of magnetic energy stored is likely to be large. The PPPL design has a maximum of 7250 megajoules ( 2000 kilowatt hours) stored in the magnetic field, of which 5000 megajoules is associated with fields cycled in normal operation. The magnetic energy in poloidal fields at the end of the ignition phase is on the order of 50,000 megajoules in the UWMAK-I design (with uncertainties depending on plasma resistivity). The change in poloidal field energy divided by maximum plausible power supply in ignition is the lower limit on ignition time and poloidal field rise time. It would appear that rise time of the poloidal magnetic field in the commercial tokamak power plant will not be much shorter than 10 seconds unless modification of the tokamak concept dramatically changes the scale. Hence an induced field of less than 5 volts/meter just outside the torus should be expected. 
D. L. Lessor

The nonionizing radiation from electric current switching and current transients can be attenuated with fairly thin layers of conducting material. It seems probable that the ionizing radiation shields and other conducting material present will be adequate to attenuate all the nonionizing radiation from its interior. The skin depth (depth for e-fold reduction, or reduction to $37 \%$ of incident field strength) for lower frequency radiation than optical is $(10)$

$$
\delta=(\pi \mu \sigma \nu)^{-1 / 2}
$$

where $v$ is frequency, $\sigma$ is material conductivity, and $\mu$ is medium permeability, all in MKS units. The skin depth of aluminum for 1000 kilohertz (middle of the AM radio band) is

$$
\delta \simeq 8 . \times 10^{-5} \text { meters }=.008 \text { centimeters } \simeq .003 \text { inch } .
$$

The electric field amplitude $E$ of this radiation falls off with distance $X$ into the medium according to

$$
E(X)=E_{0} \exp (-X / \delta)
$$

where $E_{0}$ is electric field amplitude at the surface. At a distance $2 \delta$ into the medium, the amplitude will have fallen to $\exp (-2) \simeq .14 \simeq(0.37)^{2}$ of $i$ ts value at the surface. Thus even a foil of good conductor is sufficient to attenuate this radiation, but a few inches of concrete will do so also. Microwave radiation would penetrate an even smaller distance owing to its higher frequency. The exposure of biological specimens and of radio and television receivers can be made small with reasonable convenience and cost, if indeed it is not small already from the nature of the design.

\section{Decay of Electromagnetic Fields in Good Conductors}

The objective of this development is to justify a diffusion relation for the decay of magnetic fields in a configuration having an abundance of good conductors, as in a fusion reactor. We try to show that the decay is dominated by the diffusion-like part. Consider the electromagnetic fields in the configuration of conducting material, Fourier analyzing the spatial dependence:

$$
\begin{aligned}
& \underline{E}(\underline{r}, t)=\frac{1}{(2 \pi)^{3 / 2}} \int d^{3} k \underline{E}(k, t) e^{i \underline{k} \cdot \underline{r}} \\
& \underline{H}(\underline{r}, t)=\frac{1}{(2 \pi)^{3 / 2}} \int d^{3} k \underline{H}(k, t) e^{i \underline{k} \cdot \underline{r}} \\
& |\underline{k}|<N \frac{2 \pi}{L}
\end{aligned}
$$

In a fusion Reactor, the initial amplitudes $\underline{E}(\underline{k}, 0)$ and $\underline{H}(\underline{k}, 0)$ will have large magnitudes only for 
where $N$ is a reasonably low integer and $L$ is a typical transverse dimension of a field coil or other hardware element. This is because the fields vary reasonably smoothly across the fieldrelated hardware elements. For a fusion reactor, $L$ could be on the order of 0.1 meter, and $N$ might be on the order of 20 . We are justified in the following in focusing attention on time evolution of those amplitude components $\underline{E}(\underline{k}, t)$ and $\underline{H}(\underline{k}, t)$ which satisfy relation 3 .

We will consider, instead of the evolution of field amplitudes in the actual geometry, the evolution of field components in a uniform linear medium. We then try to identify mathematical features which might be expected to persist in the actual geometry. We will describe this medium with linear constitutive relations supplementing Maxwell's equations:

$$
\begin{array}{ll}
\nabla \times \underline{H}=\underline{J}+\underline{\dot{D}} & \nabla \cdot \underline{D}=\rho \\
\nabla \times E=-\underline{\dot{B}} & \nabla \cdot \underline{B}=0 \\
\underline{B}=\mu \underline{H} & \underline{D}=\varepsilon \underline{E} \quad \underline{J}=\sigma \underline{E}
\end{array}
$$

Taking the curl of the curl $\underline{H}$ equation gives

$$
\begin{aligned}
\nabla \times(\nabla \times \underline{H}) & =\nabla \times \underline{J}+\nabla \times \underline{B} \\
& =\nabla \times \sigma \underline{E}+\nabla \times \varepsilon \underline{\underline{E}} \\
& =-\sigma \underline{B}-\varepsilon \underline{\ddot{B}} . \\
& =-\sigma \mu \underline{\dot{H}}-\varepsilon \mu \underline{H}
\end{aligned}
$$

Similarly, taking the time derivative of the curl $\underline{H}$ equation yields

$$
\begin{aligned}
& \nabla \times \underline{\dot{H}}=\underline{j}+\underline{\ddot{D}} \\
& \nabla \times(\underline{B} / \mu)=\sigma \underline{\underline{E}}+\varepsilon \underline{\underline{E}} \\
& -\frac{1}{\mu} \nabla \times(\nabla \times \underline{E})=\sigma \underline{\underline{E}}+\varepsilon \underline{\underline{E}} \\
& \nabla \times(\nabla \times \underline{E})=-\sigma \mu \underline{\dot{E}}-\varepsilon \mu \underline{E}
\end{aligned}
$$

The equations 6 and 7 obeyed by $\underline{H}$ and $\underline{E}$, respectively, are identical, but $\underline{H}$ in a linear medium satisfies the zero divergence condition

$$
\nabla \cdot \underline{H}=0,
$$

while E may have a nonzero divergence:

$$
\nabla \cdot \underline{E}=\rho / \varepsilon
$$

Hence inserting a representation of the form 2 into the equation 6 gives

$$
\varepsilon \mu \underline{H}+\sigma \mu \underline{\dot{H}}+k^{2} \underline{H}=0
$$

where we have used

$$
\underline{k} \cdot \underline{H}=0
$$


from equation 8. Here $\underline{H}$ means $\underline{H}(\underline{k}, t)$. Substituting a representation of the form 1 into the equation 7 , by contrast, gives

$$
\varepsilon \mu \underline{\ddot{E}}+\sigma \mu \underline{\underline{E}}-\underline{k} \times(\underline{k} \times \underline{E})=\underline{0}
$$

which implies

$$
\varepsilon \mu \ddot{E}_{11}+\sigma \mu \underline{\dot{E}}_{\mid 1}=\underline{0}
$$

and

$$
\varepsilon \mu \ddot{E}_{\perp}+\sigma \mu \underline{\dot{E}}_{\perp}+k^{2} \underline{E}_{\perp}=\underline{0}
$$

In equations 13 and 14 , the parallel and perpendicular symbol subscripts denote parts parallel and perpendicular, respectively, to $\underline{k}$. The vector $\underline{E}$ is $\underline{E}(\underline{k}, t)$ as defined in equation 1 . We can actually obtain a first integral of equation 13

$$
\varepsilon \mu \dot{E}_{11}+\sigma \mu \underline{E}_{11}=\underline{0}
$$

from the continuity equation

$$
\frac{\partial \rho}{\partial t}+\nabla \cdot \underline{J}=0
$$

and from the constitutive relations and Maxwell's equations. Equation 15 implies

$$
\underline{E}_{||}(\underline{k}, t)=\underline{E}_{||}\left(\underline{k}_{1}, 0\right) \exp \left(-\frac{\sigma}{\varepsilon} t\right)
$$

and hence

$$
\rho(\underline{r}, t)=\rho(\underline{r}, 0) \exp \left(-\frac{\sigma}{\varepsilon} t\right)
$$

Charge density $\rho$ and the part of the electric field which has net charge density $\rho$ as its source decays with a time constant $\varepsilon / \sigma$ in an infinite linear conducting medium. This is a fast decay in conducting materials; $\varepsilon / \sigma$ is approximately $10^{-17}$ seconds in stainless steel.

We shall see that magnetic fields in good conductors decay much more slowly in our parameter domain of interest, as implied by equation 10 . Equation 10 has solutions of the form

$$
\underline{H}(\underline{k}, t)=\underline{H}^{(1)}(\underline{k}) e^{\lambda 1} t+\underline{H}^{(2)}(\underline{k}) e^{\lambda_{2} t}
$$

while equation 14 has similar solutions:

$$
\underline{E}_{\perp}(\underline{k}, t)=\underline{E}_{\perp}^{(1)}(\underline{k}) e^{\lambda 1 t}+\underline{E}_{\perp}^{(2)}(\underline{k}) e^{\lambda_{2} t}
$$

with

$$
\lambda_{1}=-\frac{\sigma}{2 \varepsilon}+\frac{1}{2} \sqrt{\left(\frac{\sigma}{\varepsilon}\right)^{2}-\frac{4 k^{2}}{\varepsilon \mu}}
$$




$$
\lambda_{2}=-\frac{\sigma}{2 \varepsilon}-\frac{1}{2} \sqrt{\left(\frac{\sigma}{\varepsilon}\right)^{2}-\frac{4 k^{2}}{\varepsilon \mu}}
$$

We can deduce from equations 21 and 22 the condition that relaxation of an initial field configuration spatial Fourier component be overdamped with no ringing, namely

$$
\left(\frac{\sigma}{\varepsilon}\right)^{2}>\frac{4 k^{2}}{\varepsilon \mu}
$$

For most of the propagation vectors for which the initial amplitude $\underline{H}(\underline{k}, 0)$ is significant, the condition 23 will be satisfied. The amplitudes which will ring are those for which

$$
k^{2}>\frac{\mu \sigma^{2}}{4 \varepsilon}
$$

or

$$
\lambda_{\underline{k}}<\frac{4 \pi}{\sigma} \sqrt{\frac{\varepsilon}{\mu}}
$$

where spatial wavelength $\lambda_{k}$ is $2 \pi / k$.

For stainless steel we can take

$$
\begin{aligned}
\sigma & =10^{6} \text { mos } / \text { meter } \\
\varepsilon & =8.854 \times 10^{-12} \text { coulombs } / \text { joule meter } \\
\mu & =4 \pi \times 10^{-7} \text { Henries/meter },
\end{aligned}
$$

so the criterion 25 for ringing is

$$
\lambda_{\underline{k}}<3.3 \times 10^{-8} \text { meters }
$$

Since the spatial wavelength for ringing is so short compared with those needed in a Fourier representation of the initial magnetic fields, we assert that almost all the initial field components decay in overdamped fashion and relation 24 is satisfied for $k$ values of prime interest.

For good conductors we can accordingly make the approximation

$$
\begin{aligned}
\lambda_{1} & \simeq-\frac{\sigma}{2 \varepsilon}+\frac{\sigma}{2 \varepsilon}\left(1-\frac{2 k^{2} \varepsilon}{\sigma^{2} \mu}\right) \\
& \simeq-\frac{k^{2}}{\sigma \mu} \\
\lambda_{2} & \simeq-\frac{\sigma}{\varepsilon}+\frac{k^{2}}{\sigma \mu}
\end{aligned}
$$

We see that for good conductors

$$
\left|\lambda_{1}\right| \ll\left|\lambda_{2}\right|
$$


since

$$
\frac{\sigma}{\varepsilon}>>\frac{k^{2}}{\sigma r}
$$

The curl E equation implies that for our solutions 19 and 20

$$
\begin{aligned}
& \underline{i k} \times \underline{E}_{\perp}^{(1)}(\underline{k})=-\lambda_{1} \mu \underline{H}^{(1)}(\underline{k}) \\
& \underline{i k} \times \underline{E}_{\perp}^{(2)}(\underline{k})=-\lambda_{2} \mu \underline{H}^{(2)}(\underline{k})
\end{aligned}
$$

If we start at time $t=0$ with zero electric field in the conducting medium, we have from equation 20

$$
\underline{E}_{\perp}^{(1)}(\underline{k})=-E_{\perp}^{(2)}(\underline{k})
$$

Hence we can write for the magnetic field vector $H(\underline{k}, t)$ :

$$
\begin{aligned}
\underline{H}(\underline{k}, t)= & -\left(\lambda_{1} \mu\right)^{-1} \underline{i k} \times \underline{E}_{\perp}^{(1)}(\underline{k}) e^{\lambda_{1} t} \\
& +\left(\lambda_{2} \mu\right)^{-1}{ }_{i \underline{k}} \times \underline{E}_{\perp}^{(1)}(\underline{k}) e^{\lambda_{2} t}
\end{aligned}
$$

Thus the ratio of the amplitude of slow decaying component to the fast decaying component is

$$
\frac{\left|\underline{H}^{(1)}(\underline{k})\right|}{\left|\underline{H}^{(2)}(\underline{k})\right|}=\frac{\lambda_{2}}{\lambda_{1}} \simeq \frac{-\frac{\sigma}{\varepsilon}+\frac{k^{2}}{\sigma \mu}}{-\frac{k^{2}}{\sigma \mu}}>1
$$

The magnetic field Fourier components in the conducting medium decay primarily by the slow process with a time constant $\tau_{1}$ given by

$$
\tau_{1}=\left(-\lambda_{1}\right)^{-1}=\frac{\sigma \mu}{k^{2}}
$$

Since the maximum initial amplitudes $\underline{H}(\underline{k}, 0)$ occur for a $k$ value proportional to $L^{-1}$ where $L$ is a system length, we obtain a diffusion relation of the form

$$
\tau \propto \sigma \mu L^{2}
$$

for magnetic field relaxation.

With the zero electric field initial condition (relation 34), the electric field vector $\underline{k}$ Fourier component can be written

$$
\underline{E}_{\perp}\left(\underline{k}_{1} t\right)=\underline{E}_{\perp}^{(1)}(\underline{k})\left(e^{\lambda_{1} t}-e^{\lambda_{2} t}\right)
$$


which reaches maximum magnitude at a time $t_{e}$ given by

$$
t_{e}=\left(\lambda_{1}-\lambda_{2}\right)^{-1} \text { en }\left(\lambda_{2} / \lambda_{1}\right)
$$

The curl $\underline{H}$ Maxwell equation implies

$$
\underline{E}_{\perp}^{(1)}(\underline{k})=\left(\sigma+\varepsilon \lambda_{1}\right)^{-1} \underline{i} \underline{k} \times \underline{H}^{(1)}(\underline{k})
$$

Hence the maximum magnitude induced electric field Fourier component from the decay of this magnetic field Fourier component will be

$$
\left.E_{1}\left(\underline{k}, t_{e}\right)=i \underline{k} \times \underline{H}^{(1)}(\underline{k})\left(\sigma+\varepsilon \lambda_{1}\right)^{-1}\left(\frac{\lambda_{1}}{\lambda_{2}}\right)^{\left(-\frac{\lambda_{1}}{\lambda_{1}-\lambda_{2}}\right.}\right)\left(\frac{\lambda_{2}-\lambda_{1}}{\lambda_{2}}\right)
$$

Taking the approximation

$$
\frac{k^{2}}{\varepsilon \mu} \ll\left(\frac{\sigma}{\varepsilon}\right)^{2}
$$

we obtain

$$
\underline{E}_{\perp}(\underline{k}, t)=\frac{i \underline{k} \times \mu \underline{H}^{(1)}(\underline{k})}{\mu \sigma}
$$

Noting again that the $\underline{k}$ of maximum initial amplitude is proportional to $\mathrm{L}^{-1}$, we obtain

$$
\operatorname{Max}|\underline{E}| \simeq\left[\frac{\left[\frac{1}{B}(1)\right.}{\mu \sigma}\right]=\left[\frac{L B(1)}{\mu \sigma L^{2}}\right]=\left[\frac{L B(1)}{\tau}\right]
$$

where $L$ is a typical system transverse dimension.

In this development we have assumed that all space is filled with material of conductivity $\sigma$ which is on the order of metal conductivities. While there is an abundance of metal in and around a tokamak power plant, there is also much volume of low density, low conductivity material. Magnetic flux which does not penetrate conducting material is not "propped up" by eddy currents in a magnetic field failure like the flux that links conducting material. The energy associated with a magnetic field configuration in the absence of conducting material goes primarily into radiation instead of Joule heating. In a fusion power device, we would expect most of the magnetic field energy to go into Joule heating in a magnetic field failure and the magnetic field line dissipation to be a diffusive one, owing to the large amounts of highly conducting material. Consider, however, a mechanical break in a field coil with an immediate large separation. The electric field which would try to force current across the gap would be huge and it is difficult to imagine a break in a major field coil that did not result in arcing. To get some idea of the spectrum of radiation that would occur, let us now consider the opposite extreme case of the magnetic field collapsing in a region in which the conductivity is everywhere zero. 
For conductivity $\sigma=0$, the decay constants in equations 21 and 22 become

$$
\begin{aligned}
& \lambda_{1}=1 \frac{k}{\sqrt{\varepsilon \mu}}=1 \mathrm{kc} / \mathrm{n} \\
& \lambda_{2}=-1 \frac{k}{\sqrt{\varepsilon \mu}}=-1 \mathrm{kc} / \mathrm{n}
\end{aligned}
$$

where $c$ is the speed of light and $n$ is the index of refraction of the medium for radiation of wave vector $k$. From equations 32 and 33 we see that electric field amplitude components of the electric field in the collapse would be related to the magnetic field by

$$
\begin{aligned}
& i \hat{k} \times \underline{E}_{\perp}^{(1)}(\underline{k})=-i \frac{c}{n} \mu \underline{H}_{\perp}^{(1)}(\underline{k}) \\
& i \hat{k} \times \underline{E}_{\perp}^{(2)}(\underline{k})=i \frac{c}{n} \mu H_{-1}^{(2)}(\underline{k})
\end{aligned}
$$

Here $\hat{k}$ is a unit vector in the direction of propagation vector $\underline{k}$. Thus the electric field amplitude $\underline{E}(i)(\underline{k})$ will be on the order of the speed of light times the corresponding magnetic field amplitude $\underline{B}^{(i)}(\underline{k})$. With zero electric field at the start of the magnetic field collapse, the relation 39 still holds in the zero conductivity case and becomes

$$
\begin{aligned}
\underline{E}_{\perp}(\underline{k}, t) & =\underline{E}_{\perp}^{(1)}(\underline{k}) 2 i \sin \frac{k c}{n} t \\
& =\frac{c}{n} \hat{k} \times \underline{B}_{\perp}^{(1)}(\underline{k}) 2 i \sin \frac{k c}{n} t
\end{aligned}
$$

The Fourier amplitudes of the initial magnetic and electric field amplitudes for a given $\underline{k}$ contribute to a radiation pulse parallel and antiparallel to $k$. The electric field in the vicinity of an initially localized but collapsing magnetic field would rise to a value on the order of the speed of light times the initial magnetic field before the propagation along the $k$ vectors dispersed the energy. For an initial field of 10 tesla, this electric field could rise to a value on the order of $3.0 \times 10^{9} \mathrm{~V} / \mathrm{m}$ in this zero conductivity approximation. Because this electric field is roughly a thousand times breakdown field strength in atmospheric pressure air, we conclude that the assumption of negligible conductivity will never be valid in the vicinity of collapsing intense magnetic fields in fusion reactors. Arcing will always accompany a break in a major field coil, and the resulting electrical currents will let the field down in a diffusion limited way with modest radiation. The wavelengths of the modest amounts of radiation emitted from macroscopic current flows should be on the order of transverse dimensions of fusion reactor coil system elements. 


\section{MAGNETIC FIELD TRANSIENTS FROM TOKAMAK MALFUNCTIONS}

\section{L. Lessor}

We have seen that magnetic field transients in the normal operating cycle of currently envisioned tokamak power reactors are too slow to produce any new environmental hazards through their induced electric fields. We must now consider whether failure in any of the magnetic field coils can induce hazardous field transients or quasi-steady magnetic fields.

It is possible that one or more of the toroidal field coils could fail in operation, either by going normal or by having the current path broken. The resulting magnetic field distortion will possibly result in the loss of the plasma to the vacuum wall, which should do no serious damage to the walls unless it occurs with excessive frequency. The cancellation of low order multipole moments from the array of toroidal field coils will no longer hold when the current has gone to zero in the failed coil. The magnetic field transient, the forces on magnets adjacent to the failed coil, and the resulting magnetic field should be considered in the system design.

A superconducting to normal transition in one toroidal field coil will result in joule heat dissipation in that coil, with energy being extracted from the magnetic field. The currents in other superconducting loops will change to maintain constant magnetic flux through them, unless the resulting current change results in exceeding critical limits and producing superconducting to normal transition in the other coils. Assuming only one superconducting coil goes normal and has its current decay, the resulting magnetic field at points well outside the torus should be smaller in magnitude than that from the system of toroidal field coils with all but one carrying its standard operating current.

The magnetic field from the set of toroidal field coils with all but one carrying normal operating current should at points well outside the torus approximate the magnetic field from a dipole located at the position of the failed coil. The magnetic moment of this effective dipole will be the negative of the magnetic moment from normal operation of that coil. Thus the magnetic field B from the toroidal field coils with one failed at a field point distance $R$ in the direction of unit vector $\hat{r}$ from the failed coil will be

$$
\underline{B}=(3 \hat{r} \hat{r} \cdot \underline{m}-\underline{m}) / R^{3}
$$

where

$$
\underline{m}=-\frac{\mu_{0}}{4 \pi} \operatorname{NIA\hat {n}}
$$

Here $I$ is the current per conductor in the toroidal field coils, $N$ is the number of turns of conductor, $A$ is the area of a conductor turn in the toroidal field coils, and $\hat{n}$ is a unit vector normal to the plane of the field coil turns. The constant $\mu_{0}$ is $4 \pi \times 10^{-7}$ in rationalized MKS units. In the UWMAK-I design, we have for one of the D-shaped toroidal field coils 
$I=10,212$ amperes

$N=2040$

$A=145$ meter $^{2}$

Thus at a distance of 40 meters from the failed coil's center in the plane of the torus (roughly the closest point outside the biological shield in UWMAK-I) we have

$B=.0047$ tesla $=47$ gauss .

The magnetic field from the toroidal field coils after one has lost its current should actually be less than this value, since currents in adjacent coils will increase to maintain their linking flux. The failure of several adjacent toroidal field coils simultaneously would result in a larger toroidal magnetic field at the biologic shield, although it would not go up linearly with number of coils failing. Since the resulting field is on the order of or smaller than that already present from poloidal field coils, it does not appear to present special problems.

Of greater concern in magnetic field failures are the possibilities of:

(1) sudden helium vaporization from heating, resulting in destructive rupture of the helium coolant system,

(2) joule heating within a magnet or other conductor sufficient to vaporize material and possibly release radioactive or otherwise hazardous material,

(3) thermal stress rupture of magnets,

(4) electric arcing, with material vaporization and release.

The thermal stress rupture of magnets is primarily an engineering problem (and in extreme cases a safety problem) rather than an environmental one, and one that will be dealt with in magnet design. The sudden vaporization of enough volume of helium coolant in superconducting magnets to cause a pressure surge in the helium coolant system is a real possibility, an accident that will probably occur frequently. It will probably be essential to provide for this expansion with pop-out safety plugs. Catching the released helium with its tritium contamination is highly desirable. It is also desirable to provide an emergency energy dump for coils thus deprived of helium coolant.

It will be necessary in engineering design of magnetic field systems to either provide the malfunction detection logic and hardware with failure possibilities reduced to near zero, or to provide an inherently safe design.

A malfunction of one or several of the superconducting coils in a tokamak power plant will cause an induced electric field. One of Maxwell's equations implies rigorously (in rationalized MKS units) that

$$
\int \underline{E} \cdot \underline{d l}=-\int \frac{\partial \underline{B}}{\partial \tau} \cdot d \underline{S},
$$

where the line integral on the left is over any closed path and the surface integral is over any surface for which that path is a boundary. The maximum electric field magnitude $E$ that can occur in large volumes of space from collapse of part of the current system generating the field is accordingly (in MKS units) 


$$
E=\text { Order of } \frac{B b}{2 \tau}
$$

where $B$ is the magnitude of the magnetic field change, $\tau$ is a measure of the time the magnetic field takes for its change, and $b$ is a typical dimension transverse to the field of the region of major field change. Critical magnetic fields of superconductors currently assure that $B$ will be somewhat less than 20 Tesla. UWMAK-I has maximum $B$ of 6 Tesla; the PPPL design pushes safety and superconductor technology 1 imits with a maximum B of 16 Tesla. Economic considerations set $b=20$ meters as an order of magnitude of transverse dimensions in a tokamak power plant. It remains to determine the most rapid time constant for magnetic field collapse. We note that the estimate we are seeking is for maximum electric field that can occur in a large volume in a magnet failure. A higher field can occur in small regions if a nearly closed loop of conductor encircles a region of changing magnetic flux; the high electric field then occurs across the conductor gap. The resulting hazard of arcing or electrical shock to personnel is a familiar one in electrical power generation and transmission and electrical machinery work, not one unique to fusion devices The collapse of a magnetic field linking a ring of good conducting material is essentially a diffusion process for migration of magnetic field lines across the conducting material. If the distance of good conducting material to be traversed by field lines is a, the time $\tau$ to traverse it is

$$
\tau=\text { Order of }\left(\sigma \mu \mathrm{a}^{2}\right)
$$

where $\sigma$ is material conductivity and $\mu$ is the permeability (in MKS units). Hence the resulting electric field induced by the decay of the magnetic field is

$$
E=\text { Order of } \frac{B b}{2 \tau}=\text { Order of } \frac{B b}{2 \sigma \mu a^{2}}
$$

Let us take order of magnitude numbers for the superconducting magnet materials with their structural and normal conducting material in a tokamak power plant:

$$
\begin{aligned}
& a=.1 \text { meter (A typical transverse dimension of a superconducting magnet of poloidal or } \\
& \text { toroidal field coils, made of slabs of superconductor, copper, and stainless } \\
& \text { steel) }
\end{aligned}
$$

These typical numbers give

$$
\tau=\operatorname{Order} \text { of }\left(\begin{array}{l}
.01 \text { seconds } \\
.7 \text { seconds }
\end{array}\right)-\text { All stainless steel coil }
$$




$$
E=\text { Order of }\left(\begin{array}{l}
4000 \text { volts/meter } \\
70 \text { volts/meter }
\end{array}\right) \text { - All stainless steel coil }
$$

The presence of good conductor in the magnetic field slows its collapse and reduces the magnitude of the induced electric field. The electric fields indicated here are not particularly alarming, since the normal vertical electric field of the earth is 128 volts/meter over sea and 67 to 317 volts/meter over land, with electric field values of $10^{4}$ volts/meter occurring at the earth's surface under a thunder cloud. (11) Fields of the magnitudes indicated above will be much more transitory than those from a thunder. cloud. Hence the problems associated with them would appear to be safety problems, not physiological problems associated with exposure. The induced electric fields will, of course, be much smaller at distances away from the ring large compared with its diameter.

The order of magnitude argument given here for the time for magnetic field change can be supported by a calculable example. Consider a nonmagnetic conducting torus of major radius $b$ and minor radius carrying a current $I_{0}$ at time $t=0$. If the loop is normal rather than superconducting, the current will decay with a time constant $\tau=L / R$, where $R$ is the resistance of the single turn toroidal loop. The inductance $L$ and resistance $R$ of such a single turn toroid are given approximately by $(12)$

$$
\begin{aligned}
& L=\mu_{0} b[\log (8 b / a)-7 / 4] \\
& R=\frac{2 b}{\sigma a^{2}}
\end{aligned}
$$

where it has been assumed that $b$ is several times larger than $a$. Hence the time constant for decay is

$$
\tau=\left(\mu_{0} \sigma a^{2} / 2\right)[\log (8 b / a)-7 / 4] .
$$

Since the bracketed term is of order unity for a wide range of values of b/a, this time constant fits our order of magnitude estimates.

The discussion here has only by inference treated the mutual inductance between field coil loops. In a transient from a field coil failure, the magnetic flux which permeates or is encircled by other conducting material of conductivity o can diffuse out of or through that material thickness. Superconducting loops will conserve the magnetic flux through them, provided they remain superconducting. If a superconducting loop goes normal, then the flux from the other field coils linking it will no longer be "frozen in" but will diffuse out in the indicated time. There is a safety advantage in a design with magnetic field at superconductors lower than the critical value by a safety margin, so that the current increase needed to "freeze" the linking flux when other field coils fail will not cause critical field to be exceeded and a subsequent "domino effect" as current collapses in additional field coils. The alternative to such a fail safe design is an energy dumping system which switches resistive loads for energy dumping across terminals of a coil detected to be failing. Such a dumping system will probably be necessary anyway to protect the magnets from thermal stresses in a loss of coolant accident, and to reduce 
hazards of arcing that could release radioactive material. Developing emergency energy dumping systems of sufficient inherent reliability may be one significant part of the magnetics safety program, along with adequate provision for mechanical stresses. The energy dumped as Joule heat could be dissipated in a swimming pool sized pond and does not constitute a problem.

The hazard of material vaporization is a local one from electric arcing and not one of vaporization of large volumes of coil structure from Joule heating in bulk material. The energy stored in the magnetic field is likely to be sufficient to raise the temperature of the coil structure only a few hundred degrees. Consider the toroidal magnetic field at the interior of the toroid. Its magnitude $B$ will be given by

$$
B=\frac{\mu_{0}{ }^{J A} C}{2 \pi R}
$$

where $J$ is the current density in the toroidal field coils and $A_{C}$ is the total cross sectional area of toroidal field coils normal to the current. Here $A_{c}$ includes area of superconductor, substrate, and structural material, and $J$ is average current density over this composite. The toroid major radius is $R$. The energy $E$ stored in the toroidal field will be

$$
E=\frac{B^{2}}{2 \mu_{0}} \quad A_{\phi} 2 \pi R
$$

where $A_{\Phi}$ is the area threaded by the toroidal flux. The total mass $m_{c}$ of toroidal field coit material will be

$$
m_{c}=A_{c}{ }^{2} \rho^{\rho} c
$$

where $\ell_{c}$ is the length around a toroidal field coil and $p_{c}$ is its average density. Hence if all of the energy stored in all of the toroidal field is dissipated in the field coils, the energy deposited per unit field coil mass is

$$
\frac{E}{m_{c}}=\frac{B J a}{4 \rho_{c}}
$$

where we have set $A_{\Phi}=\pi a^{2}$ and $l_{C}=2 \pi a$.

Taking plausible values of $B=10$ tesla (order of magnitude set by superconductor critical fields), $\mathrm{J}=1500 \mathrm{amps} / \mathrm{cm}^{2}=1.5 \times 10^{7} \mathrm{amps} / \mathrm{meter}^{2}$ (magnitude set by superconductor technology), $\mathrm{a}=10$ meters (magnitude set by reactor economics), and $\rho_{c}=7 \mathrm{gram} / \mathrm{cm}^{3}=7000 \mathrm{kilogram} / \mathrm{meter}^{3}$, we obtain

$$
\frac{E}{m_{c}}=5.4 \times 10^{4} \text { joules } / \text { kilogram }=13 \text { calories } / \text { gram }
$$

Since the average specific heat of the field coil material is likely to be near 0.1 calorie/gram degree centigrade, a temperature rise on the order of $130^{\circ} \mathrm{C}$ is possible. This is insufficient to vaporize metal, but large volumes of helium vapor could be produced. The failure of a single toroidal field coil could produce a larger energy deposit per unit mass in the failed coil by a factor as high as two, but the temperature rise does not seem to pose problems other than the helium vaporization problem and thermal stress problems. 
A variation on the field coil failures already considered is a mechanical break that results in a wide gap opened in a field coil. One might suppose that a break in a field coil could be opened sufficiently fast and wide that the current is interrupted without arcing and that the energy associated with the failed coil's magnetic field would be dissipated by radiation. An estimate of the electric fields that would occur in such a break with intense magnetic fields gives such a high value that one is led to conclude that arcing will always occur. The resulting conduction currents will subsequently lower the fields in a diffusion limited way, since conductivity of a heavy arc will be high. Some radiation can occur, of course.

The really severe hazard associated with magnetic field failure transients in fusion reactors is the rupture of coolant and tritium breeding material enclosures, with subsequent tritium release. The conducting walls of the ductwork or other metal in the magnetic field can carry large eddy currents in a magnetic field transient and hence experience large forces. The force per unit volume $\stackrel{f}{\rightarrow}_{f}$ from eddy currents will be

$$
\underline{f}=\underline{J} \times \underline{B}
$$

where $\underline{J}$ is current density. Using the $0 \mathrm{hm}$ 's law relation

$$
\underline{\mathrm{J}}=\sigma \underline{\mathrm{S}}
$$

and the estimate of magnitude of induced electric field

$$
E=\operatorname{Order} \text { of }\left(\frac{B L}{T}\right)
$$

where $B$ is the initial magnetic field, $L$ is a typical magnetic field system transverse dimension, and $\tau$ is a time constant for magnetic field collapse, we get the estimate

$$
\operatorname{Max}\left|\underline{f}_{v}\right|=\operatorname{Order} \text { of }\left(\frac{\sigma B^{2} L}{T}\right) \text {. }
$$

A stainless steel element of conductivity $\sigma=10^{6}$ mhos/meter in a magnetic field of initial value $B=10$ tesia in a system whose intense magnetic field region had a breadth $L=10$ meters in a 0.1 second collapse could experience a force per unit volume

$$
\operatorname{Max}\left|\underline{f}_{v}\right|=\operatorname{Order} \text { of }\left(10^{10} \text { newton/meter }{ }^{3} \simeq 37,0001 \mathrm{~b} / \mathrm{in}^{3}\right)
$$

Metal items inside the magnetic field should be designed to either stand large forces or be so arranged as to break up eddy current patterns. 
1. D. D. Mahlum, Biomagnetic Effects: A Consideration in Fusion Reactor Development, BNWL-1973, Battelle, Pacific Northwest Laboratories, Richland, WA, 1976.

2. T. F. Yang, R. W. Conn, and C. W. Maynard, Stray Fields of the Conceptual Tokamak Fusion Reactor, UWMAK-I, UWFDM-64, Nuclear Eng. Department, University of Wisconsin, 1974.

3. B. Badger, et. al., (30 authors), UWMAK-I, A Wisconsin Toroidal Fusion Reactor Design, Vol. II, UWFDM-68, University of Wisconsin, May 1975.

4. R. G. Mills, Ed., A Fusion Power Plant, MATT-1050, Plasma Physics Laboratory, Princeton University, Princeton, NJ, August 1974.

5. W. C. Wolkenhauer, B. R. Leonard, A. M. Sutey, and R. W. Moir, Conceptual Design of a Fusion-Fission Hybrid Reactor Based on a Mirror Fusion Reactor with a Subcritical Gas-Cooled Fission Bianket, BNWL-SA-4865, Sec. IV, p. 18, Battelle, Pacific Northwest Laboratories, Richland, WA.

6. R. M. Bozorth, T. R. McGuire, and R. P. Hudson, "Magnetic Properties of Materials," Chap. $5 \mathrm{~g}$ of D. E. Gray, American Institute of Physics Handbook, Second Ed., McGraw-Hil1, New York, NY, 1963.

7. C. W. Allen, Astrophysical Quantities, Athlone Press, University of London, Ed. 2, Chap. 6, p. 138,1964 .

8. J. C. Slater and N. H. Frank, Electromagnetism, Eqs. (4.1-4.2), and App. V, McGraw-Hil1, New York, NY, 1947.

9. J. D. Jackson, Classical Electrodynamics, John Wiley \& Sons, Inc., New York, NY, 1962.

10. W. K. H. Panofsky and M. Phillips, Classical Electricity and Magnetism, Addison-Wesley Publishing Co., Inc., 1962.

11. R. C. Weast, Ed., Handbook of Chemistry and Physics, Chemical Rubber Co., 52nd edition, 1971-1972.

12. L. D. Landon and E. M. Lifshitz, Electrodynamics of Continuous Media, Pergamon Press, Addison-Wesley Publishing Co., Inc., Reading, MA, 1960. 


\section{OFFSITE}

A. A. Churm ERDA Chicago Patent Group 9800 S. Cass Avenue Argonne, IL 60439

J.W. Beal

ERDA Div. of Magnetic Fusion Energy

Washington, DC 20545

S. 0. Dean

ERDA Div. of Magnetic

Fusion Energy

Washington, DC 20545

E. E. Kintner

ERDA Div. of Magnetic

Fusion Energy

Washington, DC 20545

J. M. Williams

ERDA Div. of Magnetic

Fusion Energy

Washington, DC 20545

J. N. Grace

ERDA Div. of Magnetic

Fusion Energy

Washington, DC 20545

J. Baublitz

ERDA Div. of Magnetic

Fusion Energy

Washington, DC 20545

3 F. E. Coffman

ERDA Div. of Magnetic Fusion Energy

Washington, DC 20545

J. F. Decker

ERDA Div. of Magnetic

Fusion Energy

Washington, DC 20545

3 K. M. Zwilsky

ERDA Div. of Magnetic

Fusion Energy

Washington, DC 20545

Dr. Philip M. Stone

ERDA Applied Plasma Physics

Program

Washington, DC 20545

G. W. Kuswa

ERDA Div. of Laser Fusion

Washington, DC 20545
OFFSITE

R. Blaunstein

ERDA Div. of Biomedical and Environmental Research Washington, DC 20545

H. M. Busey

ERDA Div. of Military

Application

Washington, DC 20545

M. A. Bell

ERDA Div. of Safety

Standards and Compliance

Washington, DC 20545

27 ERDA Technical Information Center

M. S. Kaminsky

Argonne National Laboratory 9700 S. Cass Avenue

Argonne, IL 60439

V. A. Maroni

Argonne National Laboratory $9700 \mathrm{~S}$. Cass Avenue

Argonne, IL 60439

P. M. Persiani

Argonne National Laboratory

9700 S. Cass Avenue

Argonne, IL 60439

\section{Petrick}

Engineering and Technology Division

Argonne National Laboratory

$9700 \mathrm{~S}$. Cass Avenue

Argonne, IL 60439

W. E. Parkins, Manager

Atomics International

Component Engineering and

Technology Division

North American Rockwell

P.0. Box 309

Canoga Park, CA 91304

D. Gurinsky

Brookhaven National Laboratory

ERDA Brookhaven Area Office

Upton, NY 11973

H. J. Kouts

Brookhaven National Laboratory

ERDA Brookhaven Area Office

Upton, NY 11973

S. Pearlstein

Brookhaven National Laboratory

ERDA Brookhaven Area Office

Upton, NY 11973

\section{$\underline{\text { OFFSITE }}$}

J. R. Powell

Brookhaven National Laboratory ERDA Brookhaven Area 0ffice Upton, NY 11973

A. J. Impink, Jr. Carnegie Mellon University Pittsburgh, PA 15213

R. A. Gross

Plasma Research Laboratory

236 SW Mudd Bldg.

Columbia University

New York, NY 10027

W. C. Gough

Electric Power Research Inst.

$3412 \mathrm{Hillview}$ Ave.

Palo Alto, CA 94304

G. R. Hopkins

Gulf General Atomic

P.0. Box 1111

San Diego, CA 92112

Zeinab Sabri

Iowa State University

261 Sweeney Hall

Nuclear Engineering Department Ames, IA 50010

R. Borg

Lawrence Livermore Laboratory P. 0. Box 808

Livermore, CA 94550

T. K. Fowler

Lawrence Livermore Laboratory

P.0. Box 808

Livermore, CA 94550

R. Moir

Lawrence Livermore Laboratory

P.0. Box 808

Livermore, CA 94550

A. Carl Haussmann

Lawrence Livermore Laboratory P.0. Box 808

Livermore, CA 94550

J. Hovingh

Lawrence Livermore Laboratory

P.0. Box 808

Livermore, CA 94550

R. F. Post

Lawrence Livermore Laboratory P.O. Box 808

Livermore, CA 94550

C. J. Taylor

Lawrence Livermore Laboratory

P.0. Box 808

Livermore, CA 94550 
R. Werner

Lawrence Livermore

Laboratory

P.O. Box 808

Livermore, CA 94550

L. L. Wood

Lawrence Livermore

Laboratory

P.0. Box 808

Livermore, CA 94550

W. Bauer

Division Supervisor

of Physical Research

Sandia Labs Livermore

Livermore, CA 94550

L. Booth

Los Alamos Scientific

Laboratory

CTN Research

P.0. Box 1663

Los Alamos, NM 87544

D. J. Dudziak

Los Alamos Scientific Laboratory

CTN Research

P.0. Box 1663

Los Alamos, NM 87544

D. B. Henderson

Los Alamos Scientific

Laboratory

CTN Research

P.0. Box 1663

Los Alamos, NM 87544

E. L. Kemp

Los Alamos Scientific Laboratory

CTN Research

P.0. Box 1663

Los Alamos, NM 87544

F. L. Ribe

Los Alamos Scientific

Laboratory

CTN Research

P.0. Box 1663

Los Alamos, NM 87544

L. Stewart

Los Alamos Scientific

Laboratory

CTN Research

P.0. Box 1663

Los Alamos, NM 87544

K. Thomassen

Los Alamos Scientific

Laboratory

CTN Research

P.0. Box 1663

Los Alamos, NM 87544 $\underline{\text { OFFSITE }}$

0. K. Harling

Massachusetts Institute of

Technology

Cambridge, MA 02139

Bruno Coppi

Department of Physics

Massachusetts Institute of

Technology

Cambridge, MA 02139

L. Lidsky

Dept. of Nuclear Engineering

Massachusetts Institute of

Technology

Cambridge, MA 02139

Norm Rasmussen

Dept. of Nuclear Engineering

Massachusetts Institute of

Technology

Cambridge, MA 02139

David Rose

Massachusetts Institute of

Technology

Cambridge, MA 02139

R. E. Stickney

Mechanical Engineering

Massachusetts Institute of

Technology

Cambridge, MA 02139

J. J. Reinmann

NASA - Lewis Research Center

2100 Bookpark Rd.

Cleveland, $\mathrm{OH} \quad 44135$

Vincent Arp

National Bureau of Standards

Cryogenics Division

Boulder, CO 80302

J. F. Clarke

Oak Ridge National

Laboratory

P.0. Box Y

Oak Ridge, TN 37830

A. P. Fraas

Oak Ridge National

Laboratory

P.0. Box Y

Oak Ridge, TN 37830

J. Rand McNally, Jr.

Oak Ridge National

Laboratory

P.0. Box y

Oak Ridge, TN 37830

D. Steiner

Oak Ridge National

Laboratory

P.0. Box Y

Oak Ridge, TN 37830
OFFSITE

J. Scott

Oak Ridge National Laboratory

P. 0. Box $X$

Oak Ridge, TN 37830

J. Banford

Physics International

2700 Merced St.

San Leandro, CA 94577

R. A. Huse

Public Service Electric

and Gas Co.

80 Park Place

Newark, NJ 07101

M. Gottlieb

Princeton University, PPPL

P.0. Box 451

Princeton, NJ 08540

R. G. Mills

Princeton University

P.0. Box $45 T$

Princeton, NJ 08540

E. C. Tanner

Princeton University

P.0. Box 451

Princeton, NJ 08540

H. Perkins

Dept. of Chemistry

Princeton University

Princeton, NJ 06540

R. E. Gold

303 Sayre Hall

Forrestal Campus

P.0. Box 451

Princeton, NJ 06540

M. Kristiansen

Texas Tech. University

Lubbock, TX 79409

A. F. Haught

United Aircraft Research Lab.

United Aircraft Corporation

East Hartford, CT 06108

L. Levine

U.S. Naval Research Laboratory

Washington, DC 20390

C. Z. Serpan, Jr.

U.S. Naval Research Laboratory

Washington, DC 20390

Francis Chen

University of California

Electronics Research

Laboratory

College of Engineering

Berkeley, CA 94720 
A. J. Lichtenberg University of California Electronics Research Laboratory

College of Engineering Berkeley, CA 94720

Dave Okrent

U.C.L.A.

Los Angeles, CA 90024

C. D. Hendricks

University of Illinois

Nuclear Engineering

Laboratory

Urbana, IL 61801

G. H. Miley

University of Illinois

Nuclear Engineering

Laboratory

Urbana, IL 61801

Terry Kammash

University of Michigan

Nuclear Engineering

Department

Ann Arbor, MI 48105

Dean Abrahamson

University of Minnesota

School of Public

Affairs

Social Science

Building/309

Minneapolis, MN 55455

W. G. Davey

University of Texas

Department of Physics

Austin, TX 78712

E. Linn Draper, Jr.

University of Texas

Department of Physics

Austin, TX 78712

W. E. Drummond

University of Texas

Department of Physics

Austin, TX 78712

Abraham Hertzberg University of Washington

Aerospace Research

Laboratory

316 Guggenheim

Seattle, WA 98105

A. L. Babb

University of Washington

Nuclear Engineering

Department

Seattle, WA 98105
R. Conn

University of Wisconsin

Nuclear Engineering

Department

Madison, WI 53706

G. L. Kulcinski

University of Wisconsin

Nuclear Engineering

Department

Madison, WI 53706

C. W. Maynard

University of Wisconsin

Nuclear Engineering

Department

Madison, WI 53706

D. Lichtman

Department of Physics

University of Wisconsin

Milwaukee, WE 53201

E. E. Donaldson

Washington State University

Deparment of Physics

Pullman, WA 99163

D. D. Mahlum

Division of Biomedical

and Environmental Research

Washington, DC 20545

J. V. Vanston

Engineering Science

Building

University of Texas

Austin, TX 78712

Leslie S, Ramsey

450 North 5th Street

Indiana, PA 15701 
ERDA Richland Operations Dffice

$$
\text { W. A. Burns }
$$

Atlantic Richfield Hanford Company

\section{J. D. Kaser}

Hanford Engineering

Development Labs

D. G. Doran

H. H. Yoshikawa

\section{Battelle-Northwest}

D. T. Aase

G. S. Allison

T. W. Ambrose

D. G. Atteridge

D. A. Baker

J. L. Bates

M. A. Bayne

E. R. Bradley

J. L. Brimhall

R. L. Brodzinski

R. J. Brouns

L. R. Bunnell

L. L. Burger

S. H. Bush

N. E. Carter

T. D. Chikalla

R. G. Clark

T. L. Criswell

S. D. Dahlgren

M. T. Dana

D. E. Deonigi

R. L. Dillon

D. A. Dingee

P. J. Dionne

B. H. Duarie

J. W. Finnigan

J. C. Fox

J. J. Fuquay

J. E. Garnier

R. D. Gastil

B. F. Gore

J. N. Hartley

A. J. Haverfield

$U$. P. Jenquin

A. B. Johnson, Jr.

R. H. Jones

T. J. Kabele

W. S. Kelly

H. E. Kissinger

D. A. Kottwitz

N. Laegried

B. R. Leonard, Jr

D. L. Lessor

$10 \mathrm{H}$. B. Liemohn

R. C. Liikala

M. A. Mckinnon

R. F. Maness

R. P. Marshall
Battelle-Northwest - Continued

E. S. Murphy

R. D. Nelson

D. F. Newman

R. E. Nightingale

D. E. Olesen

L. T. Pedersen

R. T. Perry

D. R. Pratt

L. A. Rancitelli

J. F. Remark

U. S. Renné

R. E. Rhoads

W. U. Richmond

W. F. Sandusky

L. C. Schmid

N. M. Sherer

E. P. Simonen

R. I. Smith

J. K. Soldat

C. W. Stewart

K. B. Stewart

R. W. Stewart

J. A. Strand

D. L. Styris

A. M. Sutey

V. L. Teofilo

G. L. Tingey

M. T. Thomas

R. C. Thompson

L. H. Toburen

T. J. Trapp

R. Wang

R. E. Westerman

L. D. Williams

10 J. R. Young

M. G. Zimmerman

1 Technical Publications (BH)

5 Technical Information 